\title{
Analytical considerations for linear and nonlinear optimization of the theoretical minimum emittance cells: Application to the Compact Linear Collider predamping rings
}

\author{
F. Antoniou ${ }^{*}$ and Y. Papaphilippou ${ }^{\dagger}$ \\ CERN, CH-1211 Geneva 23, Switzerland \\ (Received 18 October 2013; published 23 June 2014)
}

\begin{abstract}
The theoretical minimum emittance cells are the optimal configurations for achieving the absolute minimum emittance, if specific optics constraints are satisfied at the middle of the cell's dipole. Linear lattice design options based on an analytical approach for the theoretical minimum emittance cells are presented in this paper. In particular the parametrization of the quadrupole strengths and optics functions with respect to the emittance and drift lengths is derived. A multiparametric space can be then created with all the cell parameters, from which one can choose any of them to be optimized. An application of this approach is finally presented for the linear and nonlinear optimization of the Compact Linear Collider predamping rings.
\end{abstract}

DOI: 10.1103/PhysRevSTAB.17.064002

PACS numbers: 41.85.-p, 41.75.Ht, 29.20.db

\section{INTRODUCTION}

High brilliance or luminosity requirements, for electron storage or linear colliders' damping rings, necessitate ultra low emittance beams. Under the influence of synchrotron radiation, the theoretical minimum emittance (TME) [1] is reached for specific optics conditions, including a unique high cell phase advance [2]. The strong focusing needed for accomplishing the TME conditions results in cells with intrinsically high chromaticity. The chromatic sextupoles' strengths are enhanced by the low dispersion of the TME cell and reduce the dynamic aperture (DA). The ultimate target of a low emittance cell designer is to build a compact ring, attaining a sufficiently low emittance, with an adequately large DA, driven by geometrical aperture and injection requirements. The lattice design, however, is often based on numerical tools whose optimization algorithms depend heavily on the initial conditions. Reaching the optimal solution necessitates several iterations, without necessarily having a global understanding of the interdependence between a series of optics parameters and knobs. Modern techniques, as the multiobjective genetic algorithms (MOGA) [3] or the global analysis of stable solutions (GLASS) [4] attempt to achieve a global optics optimization exploring numerically all possible solutions, within stability and performance requirements. In this paper, a different approach is followed by obtaining an analytical solution for the quadrupole strengths and a complete parametrization of

\footnotetext{
*Fanouria.Antoniou@cern.ch

Ioannis.Papaphilippou@cern.ch
}

Published by the American Physical Society under the terms of the Creative Commons Attribution 3.0 License. Further distribution of this work must maintain attribution to the author $(s)$ and the published article's title, journal citation, and DOI. the TME cell, using the thin lens approximation. In this way, all cell properties are globally determined and the optimization procedure following any design requirement can be performed in a systematic way. The properties of the cell are studied in the case of the absolute minimum emittance limit, but also away from this limit. In this respect, the terminology TME cells refers to a cell design that does not specifically reach the theoretical minimum value for the emittance. Although approximate, the obtained solutions are very close to the real thick-element optics and can be used as initial conditions for efficiently matching the lattice through numerical optics codes.

The Compact Linear Collider (CLIC) predamping rings offer an ideal test bed for applying the procedure mentioned above: they have to accommodate a large emittance beam, coming in particular from the positron source, and reduce its size to low enough values for injection into the main damping rings. The latter requirement imposes a low emittance cell linear optics design, whereas the former one necessitates a large off-momentum DA.

The paper is organized as follows: In Sec. II, the analytical expressions for the quadrupole strengths and other optics parameters of the TME cell are derived, including conditions for stability of the solutions and feasibility of the magnets. In Sec. III, the complete parametrization of the TME cells is performed using numerical examples of the analytical thinlens solutions, applicable to the CLIC predamping rings (PDR) lattice design. A validation of the method through the comparison of the results with numerical simulations using MADX [5] is presented in Sec. IV. Finally, in Sec. V an application of the analytical approach and the resonance free lattice concept [6] is used for the linear and nonlinear optimization of the CLIC PDR. 


\section{ANALYTICAL APPROACH FOR THE TME CELLS}

\section{A. The TME cell}

A schematic layout of the TME cell is displayed in Fig. 1. It consists of one dipole $\mathrm{D}$ of length $l_{d}$ and at least two families of quadrupoles $Q_{1}, Q_{2}$, as pictured. The quadrupole focal lengths are denoted by $f_{1}=1 /\left(k_{1} l_{q 1}\right)$ and $f_{2}=$ $1 /\left(k_{2} l_{q 2}\right)$ and the drifts between the elements by $s_{1}, s_{2}$ and $s_{3}$. For simplicity, the center of consecutive dipoles is considered as the entrance and exit of the TME cell.

The horizontal emittance of the beam in an isomagnetic ring:

$$
\epsilon_{x}=\frac{C_{q} \gamma^{2}}{\mathcal{J}_{x} \rho_{x}}\left\langle\mathcal{H}_{x}\right\rangle
$$

is determined by the average dispersion invariant in the dipoles, $\mathcal{H}_{x}=\gamma_{x} D_{x}^{2}+2 \alpha_{x} D_{x} D_{x}^{\prime}+\beta_{x} D_{x}^{\prime 2}$, where $\alpha_{x}, \beta_{x}, \gamma_{x}$ are the twiss parameters and $D_{x}, D_{x}^{\prime}$ the dispersion and its derivative. The parameter $C_{q}=3.84 \times 10^{-13} \mathrm{~m}$ is the quantum fluctuation coefficient for the electron, $\gamma$ the relativistic factor, $\mathcal{J}_{x}$ the damping partition number, and $\rho_{x}$ the bending radius. The minimization of the dispersion invariant average, provides the conditions of $\beta_{x}$ and $D_{x}$ at the center of the dipole, for achieving the theoretical minimum emittance [1]:

$\beta_{x \mathrm{c}}^{\min }=\frac{l_{d}}{2 \sqrt{15}}, \quad \alpha_{x \mathrm{c}}=0, \quad D_{x \mathrm{c}}^{\min }=\frac{\theta l_{d}}{24}, \quad D_{x \mathrm{c}}^{\prime}=0$,

where $\theta=\frac{l_{d}}{\rho_{x}}=\frac{2 \pi}{N_{d}}$ is the bending angle for $N_{d}$ dipoles in the ring. For a general TME cell, the geometrical emittance can be expressed as:

$$
\epsilon_{x}=\frac{C_{q} \gamma^{2}}{\mathcal{J}_{x} \rho_{x}}\left[\frac{1}{\beta_{x \mathrm{c}}}\left(D_{x \mathrm{c}}^{2}-\frac{\theta D_{x \mathrm{c}} l_{d}}{12}+\frac{\theta^{2} l_{d}^{2}}{320}\right)+\frac{\theta^{2} \beta_{x \mathrm{c}}}{12}\right],
$$

where $D_{x \mathrm{c}}$ and $\beta_{x \mathrm{c}}$ are the dispersion and beta functions at the center of the dipole. Substituting the values of $D_{x c}^{\min }$ and $\beta_{x c}^{\min }$ in Eq. (3) with their TME expressions of Eq. (2), the emittance becomes $\epsilon_{x \mathrm{TME}}=\mathcal{F} C_{q} \gamma^{3} \theta^{3}$. The scaling factor $\mathcal{F}$ for the TME lattice is $\mathcal{F}=\frac{1}{12 \sqrt{15} \mathcal{J}_{x}}$ and the damping partition number $\mathcal{J}_{x} \approx 1$, in the case of isomagnetic rings, based on dipoles without a quadrupole gradient [7].

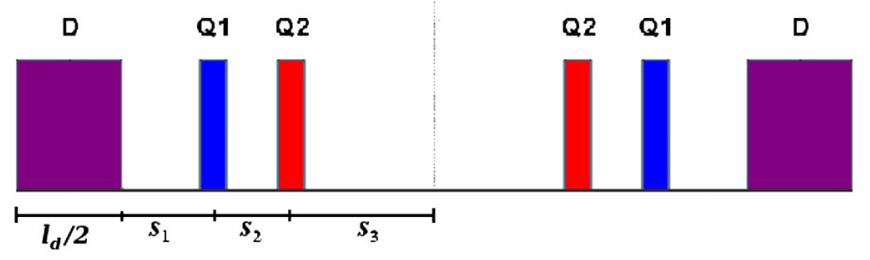

FIG. 1. Schematic layout of the TME cell.
Defining the ratios $\beta_{r}=\frac{\beta_{x c}}{\beta_{x c}^{\min }}$ and $D_{r}=\frac{D_{x c}}{D_{x c}^{\min }}$, it is useful to define the emittance detuning factor [2]:

$$
\epsilon_{r}=\frac{9+4 \beta_{r}^{2}+5\left(D_{r}-2\right) D_{r}}{8 \beta_{r}},
$$

with $\epsilon_{x}=\epsilon_{r} \cdot \epsilon_{x, \mathrm{TME}}$. The detuning factor is an indication of how much the emittance deviates from its theoretical minimum, for a given set of optics parameters at the center of the cell.

Inverting Eq. (4) and solving with respect to $\beta_{r}$, the following expression is computed:

$$
\beta_{r}=\epsilon_{r} \pm \frac{1}{2} \sqrt{-9+4 \epsilon_{r}^{2}-5\left(-2+D_{r}\right) D_{r}} .
$$

The quadratic dependence on $D_{r}$ of the argument in the square root in Eq. (5), sets an upper and a lower limit for the dispersion at the center of the dipole, in order for $\beta_{r}$ to be a real number:

$$
1-\frac{2 \sqrt{-1+\epsilon_{r}^{2}}}{\sqrt{5}} \leq D_{r} \leq 1+\frac{2 \sqrt{-1+\epsilon_{r}^{2}}}{\sqrt{5}} .
$$

\section{B. Analytical solutions for the quadrupole strengths}

The beta $\beta_{x \mathrm{c}}$ and dispersion $D_{x \mathrm{c}}$ functions, at the dipole center, impose two independent optics constraints and thus at least two quadrupole families are needed for achieving them. The horizontal optics functions are fully controlled by these two pairs of quadrupoles, whereas in the absence of additional knobs, the vertical plane optics is also uniquely defined. Using basic linear optics arguments and the thin lens approximation and for specific $\beta_{x \mathrm{c}}$ and $D_{x \mathrm{c}}$ at the center of the dipole (or $\beta_{r}$ and $D_{r}$ ), analytical expressions can be derived for the strengths of the quadrupoles:

$$
\begin{aligned}
f_{1} & =\frac{s_{2}\left(4 l_{d} s_{1}+l_{d}^{2}+8 D_{x c} \rho_{x}\right)}{\left(4 l_{d} s_{1}+l_{d}^{2}+8 D_{x c} \rho_{x}\right)+4 l_{d} s_{2}-8 D_{s} \rho_{x}} \\
& =\frac{l_{d} s_{2}\left[12 s_{1}+l_{d}\left(D_{r}+3\right)\right]}{12 l_{d}\left(s_{1}+s_{2}\right)+l_{d}^{2}\left(D_{r}+3\right)-24 D_{s} \rho_{x}}, \\
f_{2} & =-\frac{8 s_{2} D_{s} \rho_{x}}{\left(4 l_{d} s_{1}+l_{d}^{2}+8 D_{x c} \rho_{x}\right)-8 D_{s} \rho_{x}} \\
& =-\frac{24 s_{2} D_{s} \rho_{x}}{12 l_{d} s_{1}+l_{d}^{2}\left(D_{r}+3\right)-24 D_{s} \rho_{x}},
\end{aligned}
$$

which are parametrized with the drift lengths $s_{1}, s_{2}, s_{3}$. The parameter $D_{s}$ is the dispersion at the center of the cell (between two mirror symmetric quadrupoles) and is a function of the drift lengths, the optics functions at the dipole center and the bending characteristics:

$$
D_{s}=\frac{A \pm \sqrt{A^{2}+A B C}}{64 B \rho_{x}^{2}},
$$


where

$$
\begin{aligned}
A & =8 s_{2} \rho_{x}\left[l_{d}^{4}+64 D_{x \mathrm{c}}^{2} \rho_{x}^{2}+16 l_{d}^{2}\left(\beta_{x \mathrm{c}}^{2}-D_{x \mathrm{c}} \rho_{x}\right)\right] \\
& =\frac{8}{45} l_{d}^{4} s_{2}\left[12 \beta_{r}^{2}+5\left(D_{r}-3\right)^{2}\right] \rho_{x}, \\
B & =l_{d}\left(2 s_{1} l_{d}+l_{d}^{2}+8 \beta_{x \mathrm{c}}^{2}\right)-8\left(2 s_{1}+l_{d}\right) D_{x \mathrm{c}} \rho_{x} \\
& =\frac{1}{15} l_{d}^{2}\left[l_{d}\left(15+2 \beta_{r}^{2}-5 D_{r}\right)-10 s_{1}\left(D_{r}-3\right)\right], \\
C & =\frac{16 s_{3} \rho_{x}\left(4 s_{1} l_{d}+l_{d}^{2}+8 D_{x \mathrm{c}} \rho_{x}\right)}{s_{2}} \\
& =\frac{16 l_{d} s_{3}\left[12 s_{1}+l_{d}\left(D_{r}+3\right)\right] \rho_{x}}{3 s_{2}} .
\end{aligned}
$$

The calculation of $D_{s}$ springs from the symmetry requirement at the middle of the cell, $\alpha_{x}=0$. By applying the TME conditions at the middle of the dipole $\left(\alpha_{x}=0, D_{x}^{\prime}=0\right)$, the $\alpha_{x}$ function at the middle of the cell has a quadratic dependence on $\left(D_{s}^{-1}\right)$, which results in the two solutions, with opposite sign in the second component, for $D_{s}$.

The horizontal and vertical phase advances of the cell can be defined through the trace of the cell transfer matrix and from this, the horizontal phase advance can be written in a simple form as:

$\cos \mu_{x}=\frac{\left(l_{d}^{2}-8 D_{x c} \rho_{x}\right)^{2}-16 l_{d}^{2} \beta_{x \mathrm{c}}^{2}}{\left(l_{d}^{2}-8 D_{x c} \rho_{x}\right)^{2}+16 l_{d}^{2} \beta_{x \mathrm{c}}^{2}}=\frac{5\left(D_{r}-3\right)^{2}-12 \beta_{r}^{2}}{5\left(D_{r}-3\right)^{2}+12 \beta_{r}^{2}}$.

For $D_{r}=\beta_{r}=1, \mu_{x}=\arccos (1 / 4)=284.5^{\circ}$ independent on any cell parameter, which is a known property of the TME cells [2]. The expression for the vertical phase advance has a more complicated form:

$$
\begin{aligned}
\cos \mu_{y}= & 1+\frac{l_{c}}{f_{1}}+\frac{l_{c}}{f_{2}}+\frac{s_{23}\left(l_{c}-2 s_{23}\right)}{f_{1}^{2}}+\frac{s_{3}\left(l_{c}-2 s_{3}\right)}{f_{2}^{2}} \\
& +\frac{2 s_{23} l_{c}-2\left(s_{23}^{2}+s_{3}^{2}\right)}{f_{1} f_{2}}+\frac{s_{2}\left(s_{23}+s_{3}\right)\left(l_{c}-2 s_{23}\right)}{f_{1}^{2} f_{2}} \\
& +\frac{2 s_{2} s_{3}\left(l_{c}-s_{23}-s_{3}\right)}{f_{1} f_{2}^{2}}+\frac{s_{2}^{2} s_{3}\left(l_{c}-2 s_{23}\right)}{f_{1}^{2} f_{2}^{2}},
\end{aligned}
$$

where $l_{c}=l_{d}+2\left(s_{1}+s_{2}+s_{3}\right)$ the cell length and $s_{23}=s_{2}+s_{3}$. Unlike the horizontal plane, the vertical phase advance depends not only on the optics functions at the dipole center but also the cell geometry.

\section{Momentum compaction factor}

An analytical expression can also be derived for the momentum compaction factor of the cell, under the TME conditions ( $D_{x}^{\prime}=0$ at the center of the dipole), and can be written in the form:

$$
\begin{aligned}
\alpha_{p} & =\left\langle\frac{D_{x}}{\rho_{x}}\right\rangle=\frac{1}{l_{d}} \int_{0}^{l_{d}} \frac{D_{x}(s)}{\rho_{x}} d s \\
& =\frac{7}{12} \theta^{2}+\frac{2 D_{c}}{\rho_{x}}=\frac{\theta^{2}}{12}\left(D_{r}+7\right),
\end{aligned}
$$

depending only on the dipole characteristics and in particular, quite strongly on the bending angle, which explains the trend that the momentum compaction factor is reduced, when the dipoles become shorter and/or weaker. The momentum compaction factor for the absolute minimum emittance $\left(D_{r}=1\right)$ is

$$
\alpha_{p}^{\mathrm{TME}}=\frac{2 \theta^{2}}{3},
$$

which depends only on the dipole bending angle.

\section{Optics stability}

The stability criterion for both horizontal and vertical planes is

$$
\operatorname{Trace}\left(M_{x, y}\right)=2\left|\cos \mu_{x, y}\right|<2,
$$

where $M_{x, y}$ is the transfer matrix of the cell and $\mu_{x, y}$ are the horizontal and vertical phase advances per cell, respectively. The latter ensures the optics stability and can be used for constraining the cell characteristics (focal and drift lengths).

\section{E. The absolute minimum emittance limit}

In the absolute minimum emittance limit, where $\beta_{r}=D_{r}=1$, the parametric equations for the quadrupole strengths are reduced to:

$$
\begin{aligned}
& f_{1}^{\mathrm{TME}}=\frac{\left(l_{d}+3 s_{1}\right)\left(3 l_{d}+5 s_{1}\right) s_{2}}{\left(l_{d}+3 s_{1}\right)\left(3 l_{d}+5 s_{1}\right)+\left(7 l_{d}+15 s_{1}\right) s_{2} \pm 2 \sqrt{l_{d}\left(l_{d}+3 s_{1}\right)\left(3 l_{d}+5 s_{1}\right) s_{3}+l_{d}^{2} s_{2}^{2}}}, \\
& f_{2}^{\mathrm{TME}}=\frac{2 l_{d} s_{2} s_{3}}{l_{d}\left(s_{2}+2 s_{3}\right) \pm \sqrt{l_{d}\left(l_{d}+3 s_{1}\right)\left(3 l_{d}+5 s_{1}\right) s_{3}+l_{d}^{2} s_{2}^{2}}} .
\end{aligned}
$$


Applying the requirement of opposite sign quadrupole strengths in the above equations, thus $f_{1} \times f_{2}<0$, in order to assure optics stability in both planes, the case of (-) sign of Eq. (8) can be ignored. This shows that the dispersion at the symmetry point of the cell can never become negative. For the $(+)$ sign, the following constraints are derived:

$$
\begin{aligned}
& s_{3}>\frac{\left[l_{d}+3\left(s_{1}+s_{2}\right)\right]\left[3 l_{d}+5\left(s_{1}+s_{2}\right)\right]}{4 l_{d}} \text { and } \\
& s_{2} \geq \frac{\left(l_{d}+3 s_{1}\right)\left(3 l_{d}+5 s_{1}\right)}{4 l_{d}}
\end{aligned}
$$

or

$$
s_{2}+s_{3}<\frac{\left(l_{d}+3 s_{1}\right)\left(3 l_{d}+5 s_{1}\right)}{4 l_{d}}
$$

It is interesting to study the behavior of Eqs. (15) in the limit where the drift spaces lengths are going to zero. They are then reduced to:

$$
\begin{aligned}
& \left(f_{1}^{\mathrm{TME}}, f_{2}^{\mathrm{TME}}\right) \stackrel{s_{1} \rightarrow 0}{\longrightarrow}\left(\frac{3 l_{d} s_{2}}{3 l_{d}+7 s_{2}-2 \sqrt{s_{2}^{2}+3 l_{d} s_{3}}}, \frac{2 s_{2} s_{3}}{s_{2}+2 s_{3}-\sqrt{s_{2}^{2}+3 l_{d} s_{3}}}\right), \\
& \left(f_{1}^{\mathrm{TME}}, f_{2}^{\mathrm{TME}}\right) \stackrel{s_{2} \rightarrow 0}{\longrightarrow}(0,0), \\
& \left(f_{1}^{\mathrm{TME}}, f_{2}^{\mathrm{TME}}\right) \stackrel{s_{3} \rightarrow 0}{\longrightarrow}\left[\frac{s_{2}\left(3 l_{d}+5 s_{1}\right)}{3 l_{d}+5\left(s_{1}+s_{2}\right)}, \frac{4 l_{d} s_{2}^{2}}{3 l_{d}^{2}+14 l_{d} s_{1}+15 s_{1}^{2}-4 l_{d} s_{2}}\right],
\end{aligned}
$$

In the limits where $s_{1} \rightarrow 0$ or $s_{3} \rightarrow 0$ both $f_{1}$ and $f_{2}$ converge to specific values, depending on the dipole length and on the drift spaces lengths. Thus, realistic solutions exist even if the first quadrupole $Q_{1}$ is placed exactly after the dipole, without any space between them, or if the two $Q_{2}$ quadrupoles are merged to 1 . In the limit where $s_{2} \rightarrow 0$ both the focal lengths $f_{1}$ and $f_{2}$ go to zero or the quadrupole strengths to infinity. A good separation of the two quadrupoles is thus necessary in order to have a feasible TME cell. In the limit of the absolute minimum emittance and of $s_{2} \rightarrow 0$, the $\cos \phi_{y}$ function goes also to infinity verifying that those solutions are optically unstable.

\section{F. Magnet technology constraints}

Even if satisfied, the stability criteria do not necessarily guarantee technologically feasible magnet strengths. The pole tip field of the quadrupoles and chromatic sextupoles is constrained by the maximum value allowed by the chosen magnet technology. In addition, the radius of the magnets' aperture should be greater than a minimum value, defined by beam and lattice properties.

The quadrupole gradient (expressed in $[\mathrm{T} / \mathrm{m}]$ ) is defined as $g=k\left(B \rho_{x}\right)$, where $k$ is the quadrupole strength and $B \rho_{x}$ the magnetic rigidity. From the definition of the pole tip field: $B_{q}=\left.R \frac{\partial B_{y}}{\partial x}\right|_{y=0}=R g$, the gradient is $g=\frac{B_{q}}{R}$, where $R$ is the quadrupole aperture radius. Considering a circular beam pipe, the minimum required aperture radius in order to accept all the particles of the incoming beam, for a nonGaussian beam distribution, is defined by the displacement of the particles with the maximum action in the beam, defined by an emittance $\epsilon_{\max }$ and a momentum deviation $\left(\delta p / p_{0}\right)_{\max }[8]$ :

$$
R_{\min }=\sqrt{2 \beta \epsilon_{\max }}+\left(\frac{\delta p}{p_{0}}\right)_{\max } \cdot D+d_{\mathrm{co}},
$$

where $\beta$ and $D$ are the beta and dispersion functions at this location, $\left(\delta p / p_{0}\right)$ the total energy spread of the beam, and $d_{\text {co }}$ a constant reflecting the tube thickness, mechanical tolerances and maximum orbit distortion. For a Gaussian beam distribution, Eq. (17) becomes: $R_{\min }=\sqrt{2 \beta \epsilon_{\max }+\left[\left(\frac{\delta p}{p_{0}}\right)_{\max } \cdot D\right]^{2}}+d_{\mathrm{co}}$. The $R_{\min }$ can be computed for each element of the cell and takes its maximum value at the center of the quadrupoles, where the beta functions become maximum. The magnet technology constraint for the quadrupole gradient or strength is then

$$
g \leq \frac{B_{q}^{\max }}{R_{\min }} \quad \text { or } \quad \frac{1}{f l_{q}}=k \leq \frac{1}{\left(B \rho_{x}\right)} \frac{B_{q}^{\max }}{R_{\min }}
$$

In a similar way, a magnet technology constraint can be set for the sextupole strengths. As already mentioned, the TME cells are intrinsically high chromaticity cells when targeting their theoretical minimum emittance limit, as low dispersion and strong focusing are needed to achieve the ultra low emittance. The high chromaticity requires strong sextupoles for the chromaticity correction, reducing the 
dynamic aperture of the machine. The sextupoles used for the natural chromaticity correction are usually placed close to the quadrupoles, in large dispersion and beta function regions. In order to simplify the calculations, the sextupoles are considered to be placed on top of the quadrupoles, with equal lengths. The pole-tip field for the sextupoles is $B_{s}=\left(B \rho_{x}\right) b_{2} R^{2}=\left.\frac{1}{2} R^{2} \frac{\partial^{2} B_{y}}{\partial x^{2}}\right|_{y=0}$ and the sextupole gradient $\left(B \rho_{x}\right) b_{2}=B_{s} / R^{2}$. As the sextupoles are set to cancel the chromaticity induced by the quadrupoles, the sextupole strengths can be calculated by:

$$
\begin{aligned}
& \xi_{x}=-\frac{1}{4 \pi} \oint \beta_{x}\left[K_{x}(s)-S(s) D(s)\right] d s=0, \\
& \xi_{y}=-\frac{1}{4 \pi} \oint \beta_{y}\left[K_{y}(s)+S(s) D(s)\right] d s=0,
\end{aligned}
$$

where $K_{x, y}$ the focusing and defocusing quadrupole strengths and $S=\frac{b_{2}}{\left(B \rho_{x}\right)}$ the sextupole strengths. Evaluating the above integrals along the cell, the expressions for the sextupole strengths are

$$
\begin{aligned}
& S_{1}=-\frac{2 \xi_{y}^{q} \pi \beta_{x, \mathrm{~d}}+2 \xi_{x}^{q} \pi \beta_{y, \mathrm{~d}}}{l_{q} \beta_{x, \mathrm{f}} \beta_{y, \mathrm{~d}} D_{x, \mathrm{f}}-l_{q} \beta_{x, \mathrm{~d}} \beta_{y, \mathrm{f}} D_{x, \mathrm{f}}}, \\
& S_{2}=\frac{2 \xi_{y}^{q} \pi \beta_{x, \mathrm{f}}+2 \xi_{x}^{q} \pi \beta_{y, \mathrm{f}}}{l_{q} \beta_{x, \mathrm{f}} \beta_{y, \mathrm{~d}} D_{x, \mathrm{~d}}-l_{q} \beta_{x, d} \beta_{y, \mathrm{f}} D_{x, \mathrm{~d}}},
\end{aligned}
$$

where $\xi_{x, y}^{q}=-\frac{1}{4 \pi} \oint \beta_{x, y} K_{x, y} d s$ and $l_{q}$ the length of the quadrupoles. For simplicity, we consider all the quadrupoles to have the same length. In the expressions above, the index $f$ denotes the values of the optics functions on the focusing quadrupoles while $d$ denotes the values on the defocusing quadrupoles. In order to have feasible solutions, these values need to satisfy the constraint:

$$
S \leq \frac{B_{s}^{\max }}{R_{\min }^{2}} \frac{1}{\left(B \rho_{x}\right)^{2}} .
$$

Equations (5), (6), (7), (10), (18), (21) fully describe the linear optics of the TME cell. The parameter space of the cell, including geometrical and optical properties, can be determined giving the possibility to optimize the cell according to any design requirements.

\section{NUMERICAL APPLICATION}

The analytical parametrization can be used to study the performance of any TME cell of interest. Some numerical examples, applicable to the CLIC PDR lattice design, will be used to demonstrate the results. The energy of the CLIC damping rings complex of $2.86 \mathrm{GeV}$ [9] and a dipole field of $1.2 \mathrm{~T}$ are used. The required output normalized emittance from the CLIC PDR is $63 \mu \mathrm{mrad}$. Leaving a blowup margin of $10 \%$, and using Eq. (3), at least 19 dipoles (or TME cells) of $1.2 \mathrm{~T}$ field and $\theta=2 \pi / N_{d} \approx 19^{\circ}$ bending angle, are needed. The example of a TME cell with 38 dipoles of $1.2 \mathrm{~T}$ bending field and $\theta \approx 9.5^{\circ}$ bending angle is also discussed. In order to set the feasibility constraints of the quadrupole and sextupole magnets, the maximum poletip field of the quadrupoles is set to $B_{q}^{\max }=1.1 \mathrm{~T}$ [10] and for the sextupoles $B_{s}^{\max }=0.8 \mathrm{~T}$ [11], which are typical values for normal-conducting magnets. Both quadrupole and sextupole lengths are set to $l_{q}=0.3 \mathrm{~m}$. Fixing those parameters, the free parameters left are the drift space lengths, $s_{1}, s_{2}$, and $s_{3}$, and the emittance $\epsilon_{x}$, or the detuning factor $\epsilon_{r}$. The parametrization with respect to drift spaces lengths and with respect to the emittance are treated separately.

\section{A. Parametrization with the drift lengths}

At first, a constant emittance is considered and we seek the drift spaces lengths satisfying the stability constraints in both horizontal and vertical planes. By construction the horizontal plane is always optically stable, thus this constraint comes solely from the vertical plane. The vertical phase advance [defined in Eq. (11)] was calculated for all combinations of $s_{1}, s_{2}$, and $s_{3}$, for $s_{1} \in(0.5,2) \mathrm{m}$, $s_{2} \in(0.5,2) \mathrm{m}$, and $s_{3} \in(0.25,1) \mathrm{m}$. The optically stable solutions for a TME cell with bending angle of $\theta=2 \pi / 19$, corresponding to a dipole length of $l_{d}=2.6 \mathrm{~m}$ and an equilibrium emittance of $\epsilon_{x}^{\mathrm{TME}}=52 \mu \mathrm{m} \mathrm{rad}$, parametrized with $s_{1}, s_{2}$, and $s_{3}$ are shown in the left part of Fig. 2. There are two manifolds of stable solutions, clearly distinguished with respect to the vertical phase advance. Solutions above the diagonal in the $\left(s_{1}, s_{2}\right)$ plane (i.e., small $s_{1}$ and large $s_{2}$ values) correspond to small phase advances $\left(\mu_{y}<0.5\right)$, while the solutions around the diagonal correspond to large phase advances $\left(\mu_{y}>0.5\right)$. The optics stability is independent of the value of $s_{3}$, as there are stable solutions for each value of $s_{3}$, for both regions of solutions. The right part of Fig. 2 shows the stability region in the $\left(s_{1}, s_{2}\right)$ plane for different theoretical minimum emittance targets $\left(\epsilon_{\mathrm{xTME}} \sim N_{d}^{-3}\right)$, color-coded with $s_{3}$. Targeting lower TME values, only the high phase advance solutions provide optical stability.

The combinations of drift lengths satisfying the stability requirements are then applied to Eqs. (7)-(11), for the calculation of all cell properties. Figure 3 shows the parametrization of the quadrupole focal lengths (top), $f_{1}$ (left) and $f_{2}$ (right) and the horizontal (bottom, left) and vertical (bottom, right) chromaticities with the drift spaces lengths, $s_{1}, s_{2}$. The two manifolds of stable solutions are clearly distinguished with respect to the quadrupole focal lengths (especially for the defocusing quad) and the horizontal and vertical chromaticities. The small $s_{1}$-large $s_{2}$ region (low vertical phase advance solutions) corresponds to weaker quadrupole strengths and smaller chromaticities. The small to moderate $s_{2}$ region (large vertical phase advance solutions) corresponds to strong quadrupole focal lengths (especially the vertical one) and large 

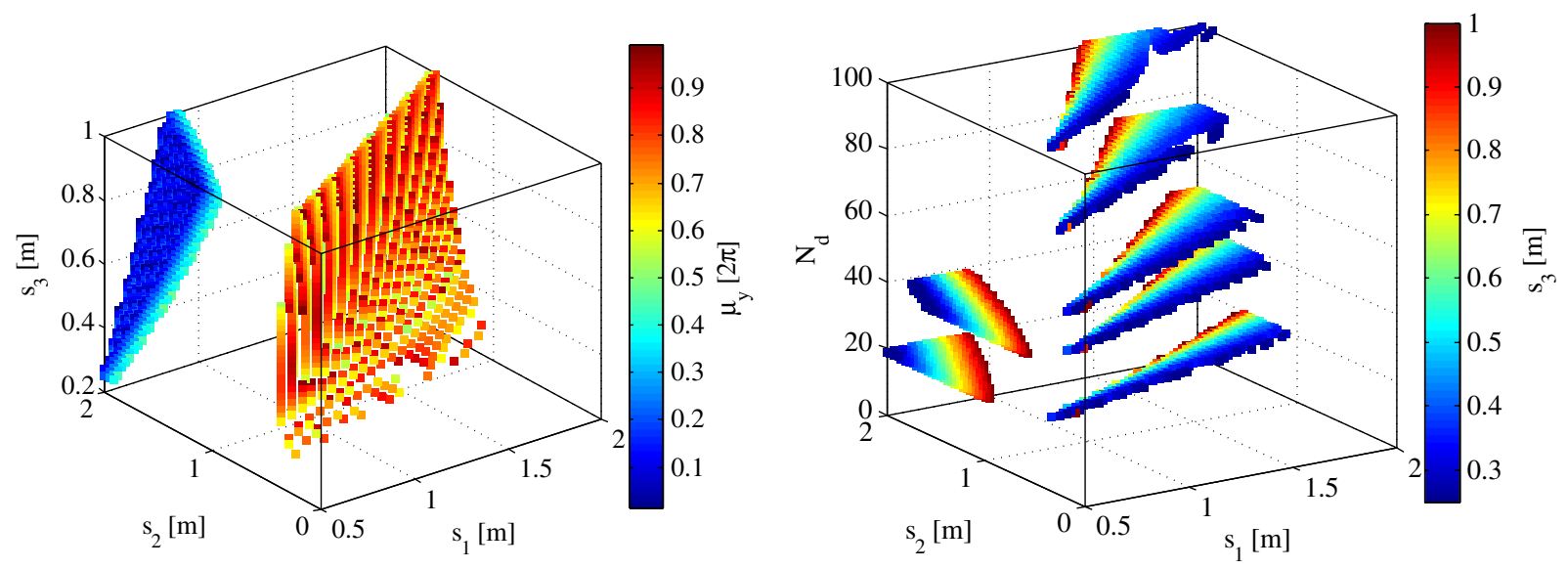

FIG. 2. Left: Parametrization of the vertical phase advance of a TME cell with a dipole bending angle of $\theta=2 \pi / 19$ with the drift spaces lengths $s_{1}, s_{2}, s_{3}$, when targeting the theoretical minimum emittance. Only solutions providing optical stability are presented. Right: The stability region in the $\left(s_{1}, s_{2}\right)$ plane for different theoretical minimum emittance targets $\left(\epsilon_{\mathrm{xTME}} \sim N_{d}^{-3}\right)$, color-coded with $s_{3}$.
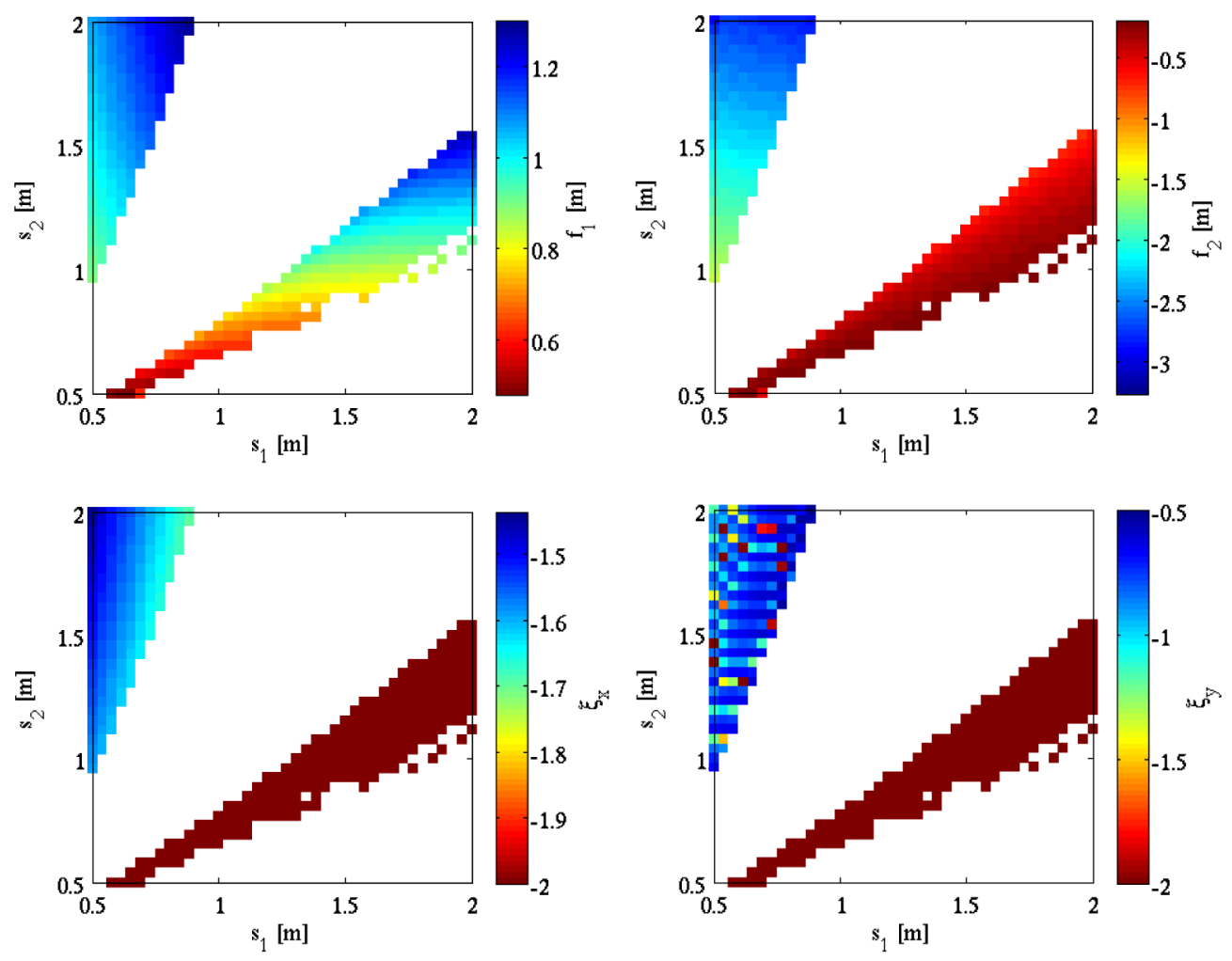

FIG. 3. Parametrization of the quadrupole focal lengths (top), $f_{1}$ (left) and $f_{2}$ (right) and the horizontal (bottom, left) and vertical (bottom, right) chromaticities with the drift spaces lengths, $s_{1}, s_{2}$ providing optical stability.

chromaticities. If magnet feasibility constraints are applied to these solutions, the latter region is rejected. The above observations can lead to the conclusion that proper choice of the drift spaces lengths triplet $\left(s_{1}, s_{2}, s_{3}\right)$ can assure the stability of the motion, and leads to the minimization of the quadrupole strengths (maximum focal lengths) and to the minimization of the cell chromaticities in both planes, achieving always the same minimum emittance.
In the case of the low emittance rings lattice design, low chromaticity solutions are of interest for larger dynamic aperture. The cell length, on the other hand, is preferred to stay as compact as possible to minimize the circumference of the ring. Figure 4 shows the $\left(s_{1}, s_{2}, s_{3}\right)$ triplets for which the absolute chromaticity in both planes is less than 2 , color-coded with the total cell length $l_{c}$ (left). In the right part of the figure, the projection of the solutions onto the 

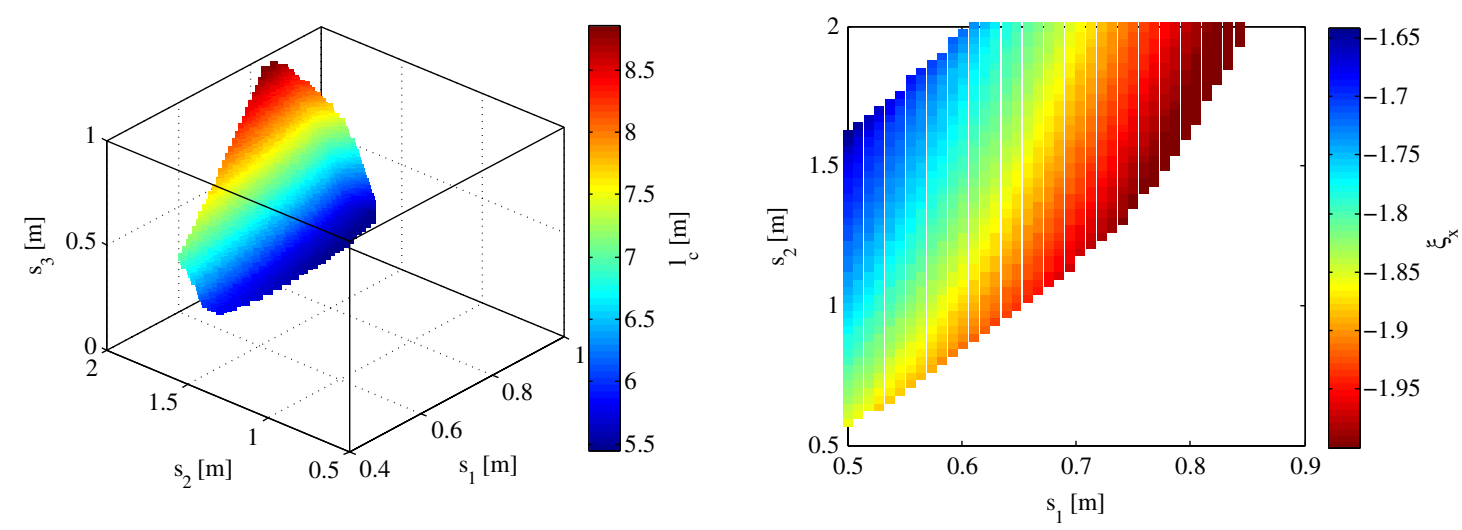

FIG. 4. Left: The $\left(s_{1}, s_{2}, s_{3}\right)$ combinations that provide the lowest chromaticity in both planes $\left(\xi_{x, y} \leq-2\right)$, color-coded with the total length of the cell. Right: Projection of the low chromaticity solutions onto the $\left(s_{1}, s_{2}\right)$ plane.

$\left(s_{1}, s_{2}\right)$ plane is shown, color-coded with the horizontal chromaticity. In order to keep the chromaticity low and the cell length as compact as possible, small values of $s_{1}$ and small to moderate values of $s_{2}$ are needed. However, even the minimum possible chromaticity of this cell is quite large $\left(\xi_{x} \sim-1.65\right)$.

\section{B. Parametrization with the emittance}

Having the drift lengths fixed, Eq. (7) combined with Eqs. (5) and (6) are studied numerically for different detuning factors $\epsilon_{r}$. In this example, the dipole bending angle is set to $\theta=2 \pi / 38$ and the drift lengths to $s_{1}=0.9 \mathrm{~m}, s_{2}=0.6 \mathrm{~m}$, and $s_{3}=0.5 \mathrm{~m}$. This configuration was found to be the optimal one for the CLIC PDR lattice design, as will be shown later.

In order to achieve the absolute minimum emittance, only one pair of initial optics functions $\left(D_{x \mathrm{c}}, \beta_{x \mathrm{c}}\right)$ or $\left(D_{r}, \beta_{r}\right)$ exists [2]. However, relaxing this requirement and detuning the cell to higher emittance values $\left(\epsilon_{r}>1\right)$, several pairs of $\left(D_{x c}, \beta_{x c}\right)$ lying in elliptical curves can achieve the same emittance, as shown by Eq. (3). Figure 5 (left) shows the solutions of $\left(D_{r}, \beta_{r}\right)$ color-coded with the detuning factor $\epsilon_{r}$. Even though, by definition, all solutions are stable in the horizontal plane, only a small fraction of them satisfy the stability criteria of the vertical plane (black squares). The parametrization of the focusing strengths with the emittance is displayed in Fig. 5 (right), with the same color-convention as before. The $f_{1}, f_{2}$ pairs for the same detuning factor lie in distorted ellipses, which get more distorted while moving to high detuning factors. In order to tune the cell to the very low emittance, strong quadrupole strengths are needed and only one combination of $\left(f_{1}, f_{2}\right)$ can tune the cell to the absolute emittance minimum. Moving away from the minimum emittance regime, the quadrupole strengths are relaxed for detuning factors greater than 2. In the upper left corner of the plot, solutions with both $f_{1}$ and $f_{2}$ positive cannot provide stability as they always provide defocusing in the vertical plane. It is interesting to notice that by changing the values of $f_{1}$ and/or $f_{2}$ by a small amount, the system remains stable if tuned in the relaxed $\epsilon_{r}$ regime but can easily get
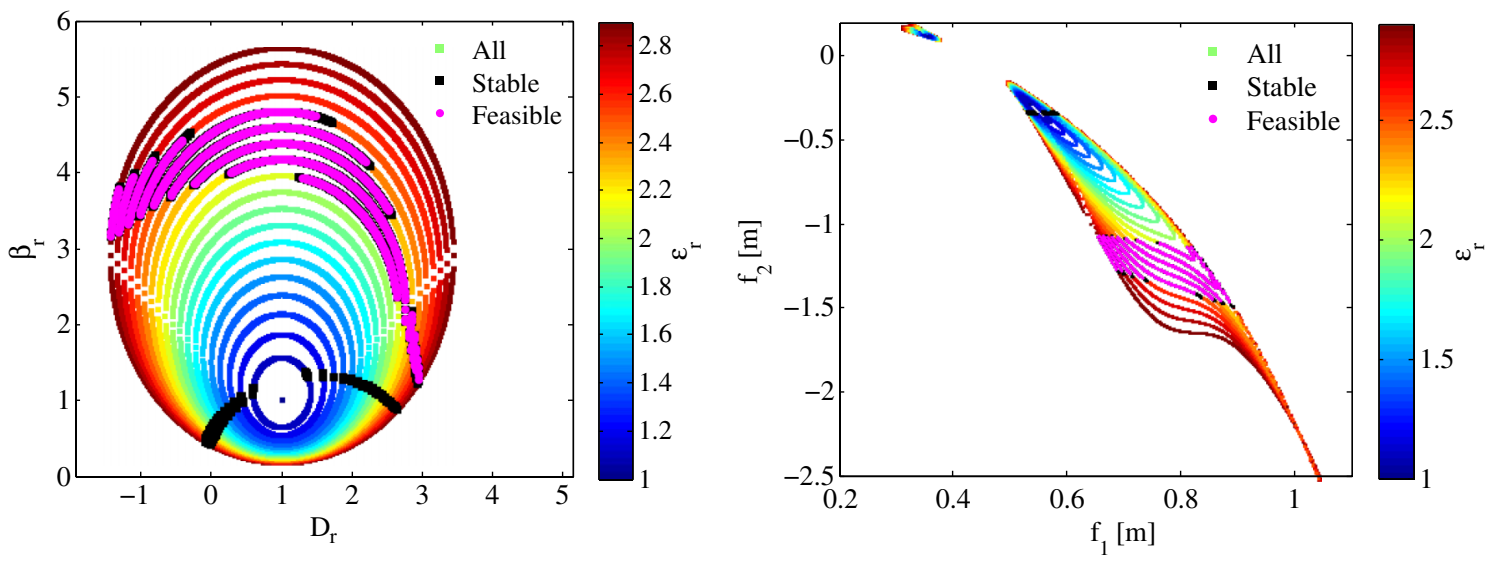

FIG. 5. Parametrization of the relative horizontal beta $\beta_{r}$ and relative dispersion $D_{r}$ at the center of the dipole (left) and the quadrupole focal lengths (right), with the cell detuning factor $\epsilon_{r}$. The stable solutions are indicated with the black squares, while the stable and feasible ones with the magenta triangles. 

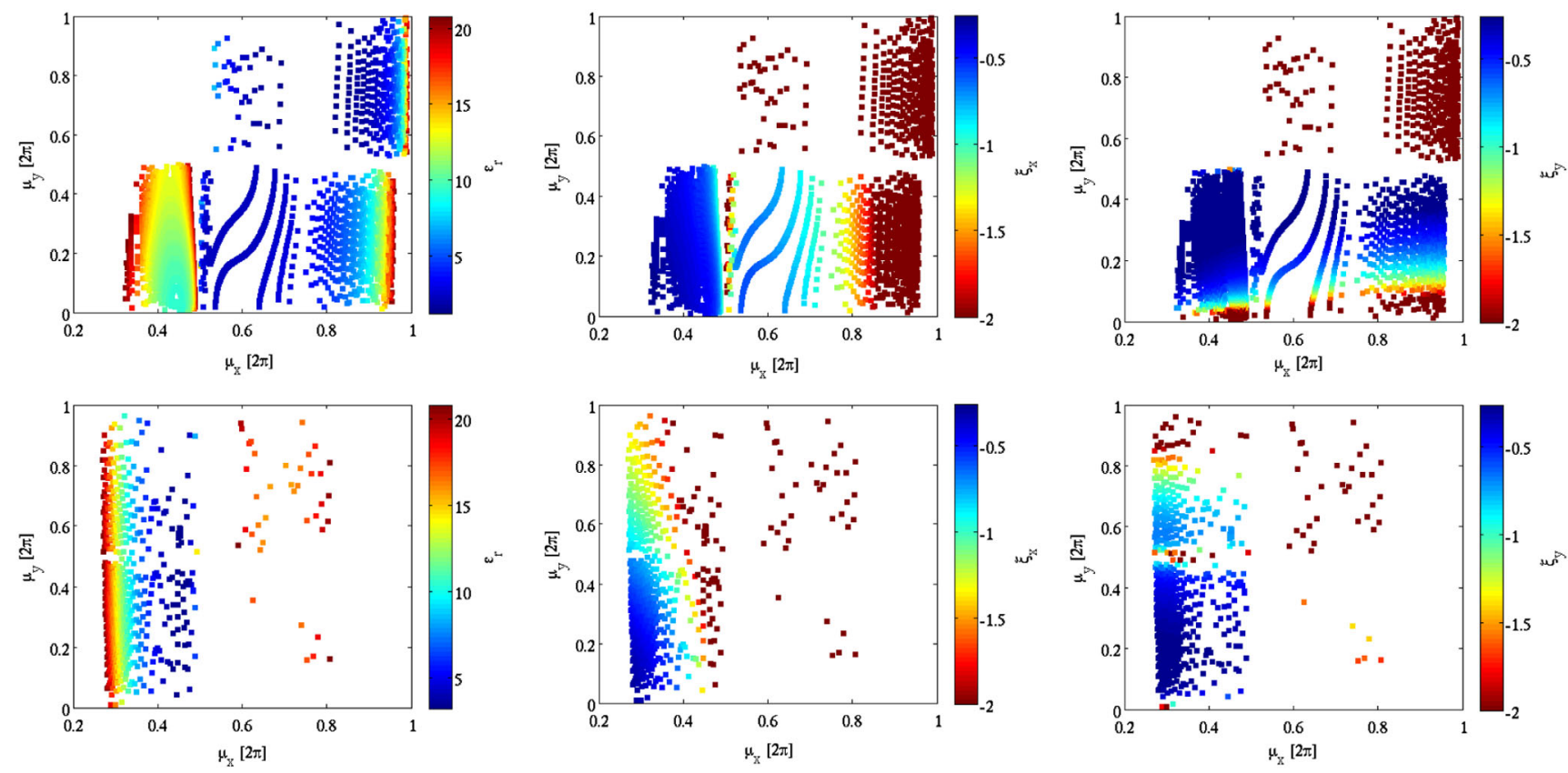

FIG. 6. Parametrization of the cell detuning factor $\epsilon_{r}$ (left), and the horizontal (middle) and vertical (right) chromaticities with the horizontal and vertical phase advances of the cell is presented for each case, for a conventional (top) and a modified (bottom) TME cell.

unstable if tuned to the absolute minimum emittance conditions.

Scanning in a broader range of the detuning factor, two different types of solutions survive the stability criteria. Solutions with focusing $Q_{1}$ and defocusing $Q_{2}$ are presented in the top part of Fig. 6, while the opposite case is presented in the bottom. Following the convention of [12], we will refer to the former case as a conventional TME cell while the latter as a modified TME cell. The parametrization of the cell detuning factor $\epsilon_{r}$ (left), and the horizontal (middle) and vertical (right) chromaticities with the horizontal and vertical phase advances of the cell is presented for each case. For a conventional TME cell the chromaticities get minimized in both planes toward small phase advances, while the emittance detuning factor becomes large. Large phase advances correspond to high chromaticity values and small detuning factors. It is interesting to notice that the high detuning factor solutions at large horizontal phase advances produce large chromaticities, as they correspond to minimum dispersion and beta functions at the center of the dipole which require strong focusing. In the case of the modified TME cell, the chromaticities are minimized for small phase advances as well, however in this case solutions with small detuning factors also exist. This type of cell is discussed in detail in [12].

\section{VALIDATION OF THE METHOD}

The results of the analytical solution were compared to numerical simulations with MADX [5] for the thin and thick lens cases. The plots of Fig. 7 show this comparison for three different values of the quadrupole lengths, $l_{q}=1,10$, and $20 \mathrm{~cm}$. The three black curves in Fig. 7 represent three different detuning factors, $\epsilon_{r}=1,1.5$, and 2 . The analytical solutions are shown in black, the solutions satisfying the stability criteria in red while the MADX solutions are presented in green, blue, and ciel, respectively. The agreement for the thin lens is excellent, demonstrating the validity of the analytical calculations. It is very interesting that, even in the thick lens case, the agreement is still very good. The analytical solution can be a very good approximation of the simulation results and can be helpful for the lattice optimization and understanding. In this way the

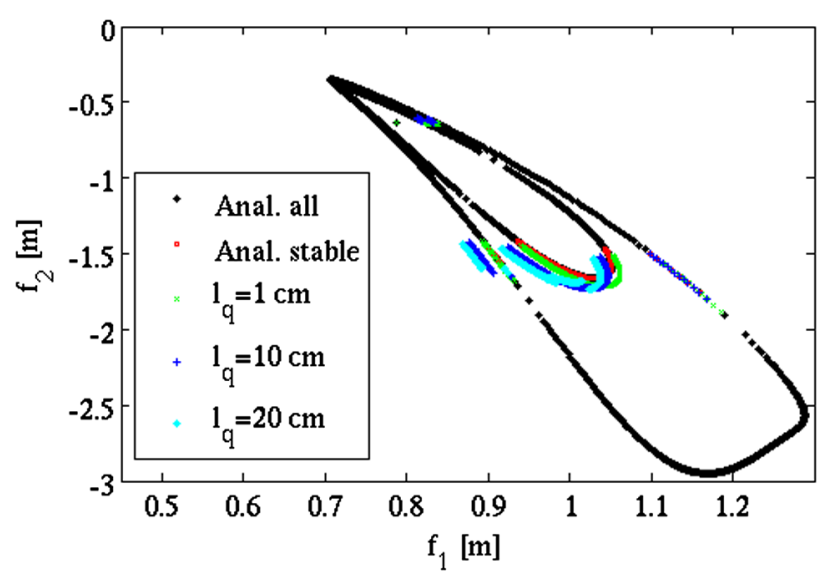

FIG. 7. Comparison between the analytical solution and MADX simulations. The analytical solutions are presented in black, the solutions satisfying the stability requirements in red and the results from MADX for different quadrupole lengths: $l_{q}=1 \mathrm{~cm}$ in green, $l_{q}=10 \mathrm{~cm}$ in blue, and $l_{q}=20 \mathrm{~cm}$ in ciel. 
optimal dipole characteristics, the geometrical characteristics of the cell and the interesting phase advances can be defined. It can also be very useful for the definition of initial conditions to be used for the lattice design using numerical tools, whose optimization algorithms depend heavily on the initial values.

This approach was thus used in order to define the optimal configuration and working point of the TME arc cells of the CLIC PDR lattice design.

\section{APPLICATION TO THE CLIC PDR DESIGN}

The CLIC predamping rings provide the first stage of damping of the $e^{+} / e^{-}$beams of the linear collider. They have to accommodate a $2.86 \mathrm{GeV}$ beam with a large input emittance of $7 \mathrm{~mm}$ rad, for positrons [13], and damp it down to a normalized emittance of $63 \mu \mathrm{m}$ rad for injection into the main DR. The required input and output parameters are given in Table I, for both electrons and positrons [14].

Unlike the DR, the PDR lattice design is not driven by the emittance requirements [9]. The large energy spread and beam size of the injected beam, especially the one coming from the positron source, impose the requirements of large momentum acceptance and dynamic aperture. Thus the PDR lattice design is focused on the dynamic aperture optimization, providing at the same time a large enough momentum acceptance and the required output emittance. Due to the more difficult characteristics of the positron beam, emphasis is given to the design of the positron predamping ring. The rings were chosen to have a racetrack configuration with two arc sections and two long straight sections (LSS), as a racetrack shape is the most compact one if only 2 dispersion free regions are required, which is valid for the case of the CLIC PDRs. The arc sections are composed by TME cells, being the most compact low emittance cells. On the other hand, the LSS are composed by FODO cells filled with damping wigglers [9]. The damping wigglers are necessary to achieve the low emittance within a fast damping time, in order to fit into the $50 \mathrm{~Hz}$ repetition rate of the collider. Permanent magnet wigglers of $B_{w}=1.9 \mathrm{~T}$ peak field and $\lambda_{w}=5 \mathrm{~cm}$ period

TABLE I. Parameters before the injection to the predamping rings and before the injection to the main damping rings.

\begin{tabular}{lccc}
\hline \hline & \multicolumn{2}{c}{ Injected } & \multirow{2}{*}{ Extracted } \\
\cline { 2 - 3 } Parameters & $e^{-}$ & $e^{+}$ & $e^{-} / e^{+}$ \\
\hline Bunch population $\left[10^{9}\right]$ & 4.7 & 6.4 & 4.4 \\
Bunch spacing [ns] & $0.5 / 1$ & $0.5 / 1$ & 0.5 \\
Bunches/train & $312 / 156$ & $312 / 156$ & $312 / 156$ \\
Number of trains & $1 / 2$ & $1 / 2$ & $1 / 2$ \\
Repetition rate $[\mathrm{Hz}]$ & 50 & 50 & 50 \\
Norm. horiz. emittance $[\mu \mathrm{m} \mathrm{rad}]$ & 100 & $7 \times 10^{3}$ & 63 \\
Norm. vert. emittance $[\mu \mathrm{m} \mathrm{rad}]$ & 100 & $7 \times 10^{3}$ & 1.5 \\
Norm. long. emittance $[\mathrm{keV} \mathrm{m}]$ & 2.86 & 2288 & 143 \\
\hline \hline
\end{tabular}

provide the fast damping time and the required output emittance [15].

For the optimization of the CLIC PDR lattice design low chromaticity solutions are of interest, as the design is focused on the dynamic aperture optimization. In this case, it is thus preferable to choose a cell that can achieve an absolute minimum emittance much lower than the requirement of the design and detune it to large detuning factors (low phase advances), in order to minimize the chromaticity. A scan on the drift spaces lengths can then be performed in order to find the optimal configuration of the cell. Here, the example of a detuned cell is considered for a dipole bending angle of $\theta=2 \pi / 38$, corresponding to a minimum emittance of $6.5 \mu \mathrm{mrad}$ and for a detuning factor of $\epsilon_{r}=10$. The emittance that this cell achieves, even if detuned by a factor of 10 , is still within the requirements of the PDR design. Unlike the case of the absolute minimum emittance described in Sec. III A, in this case all choices of $\left(s_{1}, s_{2}, s_{3}\right)$ triplets ensure optical stability. The parametrization of the horizontal (left) and vertical (right) chromaticity with the drift spaces lengths $s_{1}, s_{2}$ and the horizontal dispersion at the middle of the dipole is presented in Fig. 8. Only low chromaticity solutions for which $\left|\xi_{x, y}\right|<2$, are presented. There is a clear correlation between the $D_{x \mathrm{c}}$ and the horizontal chromaticity $\xi_{x}$. High chromaticity values correspond to negative or small dispersion at the middle of the dipole, as the latest require strong focusing by the quadrupoles. Higher dispersion values at the middle of the dipole correspond to smaller chromaticity values of the cell. For the vertical chromaticity on the other hand, the same pattern is followed for all dispersion planes, with smaller $s_{2}$ values providing the lowest vertical chromaticity. Finally, the optimal configuration of the drift spaces lengths, for the example under study, in order to provide low chromaticity in a relatively short cell, was chosen to be $\left(s_{1}, s_{2}, s_{3}\right)=(0.9,0.6,0.5) \mathrm{m}$.

\section{A. Nonlinear optimization}

The main limitation of the DA in the low emittance lattices comes from the nonlinear effects induced by the strong sextupole strengths, which are introduced for the chromaticity correction. From the nonlinear dynamics theory [16], a resonance of order $n$ defined by $n_{x} q_{x}+n_{y} q_{y}=p$, with $\left|n_{x}\right|+\left|n_{y}\right|=n$ the order of the resonance and $p$ any integer, is associated with a driving term. Based on [6], the driving term of a resonance associated with the ensemble of $N_{c}$ cells vanishes, if the resonance amplification factor is zero:

$$
\left|\sum_{p=0}^{N_{c}-1} e^{\mathrm{ip}\left(n_{x} \mu_{x, c}+n_{y} \mu_{y, c}\right)}\right|=\sqrt{\frac{1-\cos \left[N_{c}\left(n_{x} \mu_{x, c}+n_{y} \mu_{y, c}\right)\right]}{1-\cos \left(n_{x} \mu_{x, c}+n_{y} \mu_{y, c}\right)}}=0 .
$$



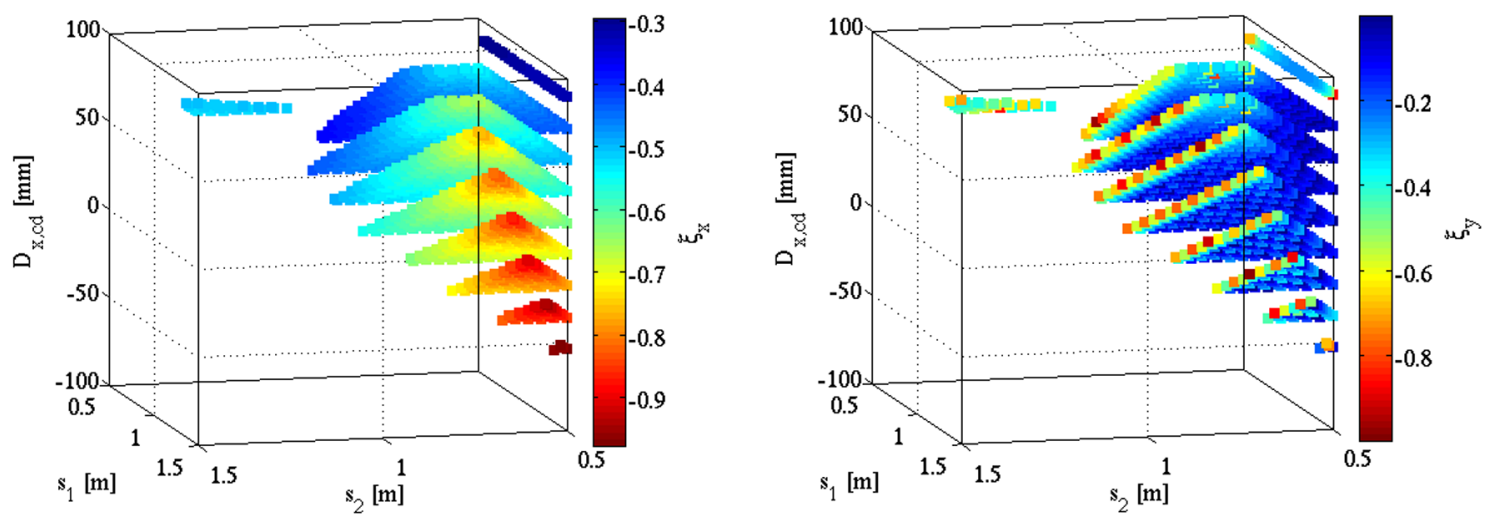

FIG. 8. Parametrization of the horizontal (left) and vertical (right) chromaticity with the drift spaces lengths $s_{1}$ and $s_{2}$ and the horizontal dispersion at the middle of the dipole, for a detuned cell $\left(\epsilon_{r}=10\right)$.

This is achieved if: $N_{c}\left(n_{x} \mu_{x, c}+n_{y} \mu_{y, c}\right)=2 k \pi$, provided the denominator of Eq. (22) is nonzero, i.e., $n_{x} \mu_{x, c}+n_{y} \mu_{y, c} \neq 2 k^{\prime} \pi$, with $k$ and $k^{\prime}$ any integers. From this, a part of a circular accelerator will not contribute to the excitation of any nonlinear resonances, except those defined by $\eta_{x} \mu_{x}+\eta_{y} \mu_{y}=2 k_{3} \pi$, if the phase advances per cell satisfy the conditions: $N_{c} \mu_{x}=2 k_{1} \pi$ and $N_{c} \mu_{y}=2 k_{2} \pi$, where $k_{1}, k_{2}$, and $k_{3}$ are any integers. Prime numbers for $N_{c}$, which in our case is the number of TME cells per arc, are interesting, as there are fewer resonances satisfying both diophantine conditions simultaneously.

The nonlinear optimization of the CLIC PDR lattice was based on the resonance free lattice concept, described above. From Eq. (3) and using a dipole field of $B_{d}=1.2 \mathrm{~T}$, at least 19 dipoles are needed in order to achieve the required output emittance. From this, convenient numbers of $N_{c}$ (number of dipoles per arc) are 11, 13, and 17, which means 26, 30, and 38 dipoles in the ring, respectively, including the dispersion suppressors' dipole. Following the results from the analytical parametrization of the TME cells, small horizontal and vertical phase advances and large detuning factors are favorable, for low cell chromaticity. The largest number of cells is better for increasing the detuning factor between the required and the minimum emittance and the cancellation of a larger number of resonance driving terms. Finally, the option of $N_{c}=38$ was chosen.

For the calculation of the resonance driving terms, the PTC-normal module of the MADX code is used, taking into account dipole and quadrupole fringe fields. The calculations are performed for different phase advances of the TME cell, while the resonance driving terms are calculated, for all the lattice. Figure 9 shows the dependence of the third order resonance driving terms, for which $(j-k) \mu_{x}+$ $(l-m) \mu_{y}=n$ and $|j-k|+|l-m|=3$, on the horizontal and vertical phase advances of the TME cell. Blue regions
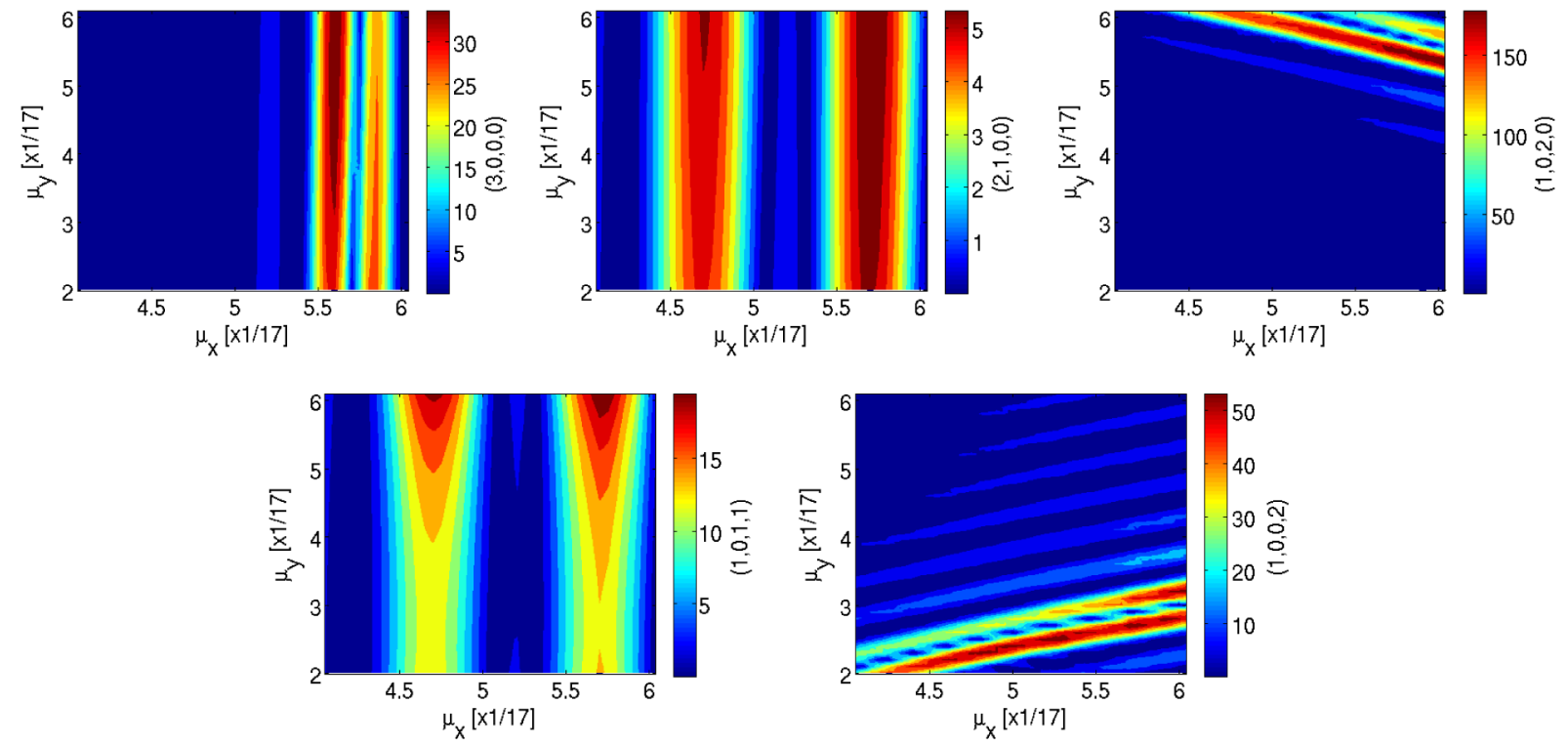

FIG. 9. Horizontal and vertical phase advances of the PDR TME cell, parametrized with the third order Hamiltonian amplitudes. 
correspond to small resonance excitation, while red regions indicate maximum excitation. Comparing the 5 Hamiltonian resonant coefficients, the $(2,1,0,0)$ mode is almost suppressed (a factor of 4-6 smaller than the other modes) and the $(1,0,1,1)$ mode is weakly excited. The nonlinear coupling term $(1,0,2,0)$ is excited at high horizontal or high vertical phase advances. The horizontal mode $(3,0,0,0)$ is also excited at high horizontal phase advances. In all cases, minimum excitation is observed, for integer multiples of $1 / 17$.

Due to the fact that strong sextupoles are introduced in the PDR lattice, for the chromaticity correction, higher order resonances should also be considered. Figure 10 shows the dependence of the fourth order resonance driving terms, for which $|j-k|+|l-m|=4$, on the horizontal and vertical phase advances of the TME cell. Maximum excitation is observed for the nonlinear coupling terms $(2,0,0,2),(2,0,2,0),(1,1,2,0)$, and $(2,0,1,1)$, especially at the high horizontal or high vertical phase advance limit of the scan. The horizontal modes $(4,0,0,0)$ and $(3,1,0,0)$ are weakly excited with respect to the other modes. The vertical modes $(0,0,4,0)$ and $(0,0,3,1)$ are also excited, in the high horizontal phase advance limit for the first and in the high vertical phase advance limit for the second. All resonance driving terms are suppressed, for phase advances that are integer multiples of $1 / 17$, as expected.

Here, the resonance driving terms are presented and discussed only to demonstrate the proof of principle of the resonance free lattice concept. In a further nonlinear optimization of the lattice, especially when high-order magnet errors are included, additional families of sextupoles, in nondispersive areas, can be used for the minimization of the resonance driving terms which limit the dynamic aperture.

Another quantity that has to be taken into account, is the amplitude dependent tune shift $\delta q_{x, y} / \delta J_{x, y}$. From first order perturbation theory, the leading order tune shift can be represented by [17]:

$$
\left(\begin{array}{c}
\delta q_{x} \\
\delta q_{y}
\end{array}\right)=\left(\begin{array}{cc}
\alpha_{\mathrm{hh}} & \alpha_{\mathrm{hv}} \\
\alpha_{\mathrm{vh}} & \alpha_{\mathrm{vv}}
\end{array}\right)\left(\begin{array}{c}
2 J_{x} \\
2 J_{y}
\end{array}\right)
$$

where $\alpha_{i j}$ are called the normalized anharmonicities and they describe the variation of the tune at different amplitudes (or action).
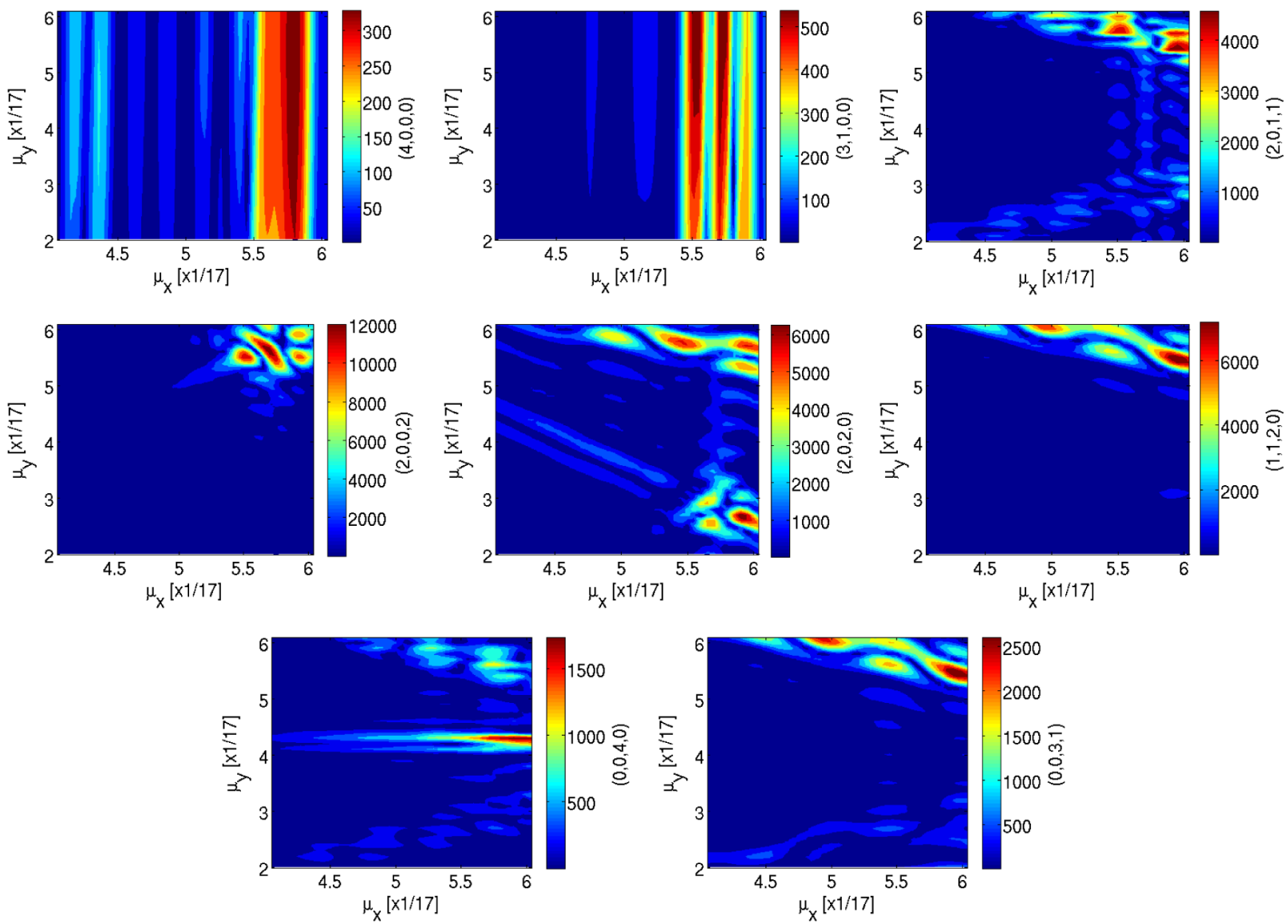

FIG. 10. Horizontal and vertical phase advances of the PDR TME cell parametrized with the fourth order Hamiltonian amplitudes. 

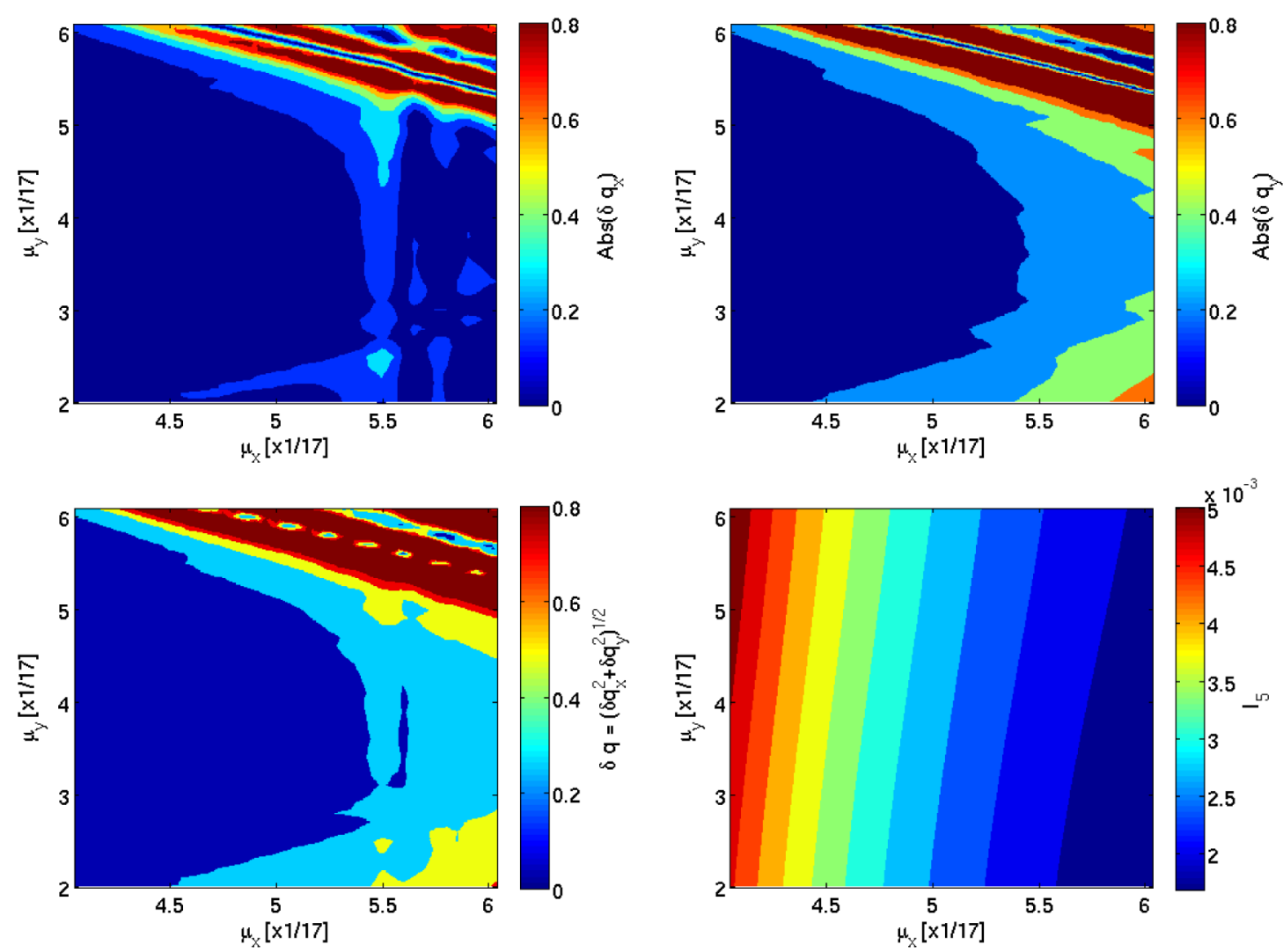

FIG. 11. Top: Parametrization of the horizontal (top, left) and vertical (top, right) amplitude dependent tune shift, with the horizontal and vertical phase advances of the TME cell. Bottom: Parametrization of the square root of the quadratic sum of the horizontal and vertical amplitude dependent tune shifts (left) and of the fifth radiation integral (right), with the horizontal and vertical phase advances of the TME cell.

Figure 11 shows the dependence of the horizontal (top, left) and vertical (top, right) detuning with amplitude, $\delta q_{x}$ and $\delta q_{y}$, respectively, on the horizontal and vertical phase advances. The bottom plots show the parametrization of the factor $\delta q=\sqrt{\delta q_{x}^{2}+\delta q_{y}^{2}}$ (left) and the fifth radiation integral $I_{5}$ (right), which is an equivalent to the horizontal emittance, with the horizontal and vertical phase advances. The amplitude dependent tune shift gets larger for large phase advances, while the emittance follows the opposite behavior. For this reason a compromise solution is chosen, where the horizontal emittance is reached for a small (but not minimum) detuning with amplitude. The optimal solution was chosen to be $\mu_{x}=5 / 17$ and $\mu_{y}=3 / 17$. With this choice, a compromise is achieved, for exciting the smallest number of resonances while maintaining a rather small amplitude detuning and chromaticity, and staying within the output emittance requirements of the design.

However, numerology shows that for this choice of phase advances, the nonlinear fifth order coupling resonance driving terms are excited, for $|j-k|=1$ and $|l-m|=4$. In this case, $\mu_{x}+4 \mu_{y}=5 / 17+4 \times 5 / 17=1$. The five modes are presented in Fig. 12, with the $(1,0,1,3)$ mode being the dominant for $\mu_{x}=5 / 17$ and $\mu_{y}=3 / 17$. The other terms get excited for higher vertical phase advances.

For the chromaticity correction, four families of sextupoles are used. A set of sextupoles are located before the focusing quadrupoles of the TME cells and a set of sextupoles after the defocusing ones. The same setup is followed for the two other families of sextupoles, which are placed in the half TME cells of the dispersion suppressors. As those sextupoles are not placed in dispersive areas, they do not contribute to the chromaticity correction, but they can be used for further nonlinear optimization of the lattice.

The optical functions of the TME cell are shown in Fig. 13, where the horizontal (black) and vertical (red) beta functions and the horizontal dispersion (green) along the cell are depicted.

The change in the particles betatron frequencies, due to the nonlinearities of the accelerator, can lead to the crossing of resonance lines in the tune diagram. This results in beam emittance blowup or in beam loss, thus, a careful choice of the betatron tunes of the linear lattice is very important for the beam quality and the beam lifetime. In the CLIC PDR lattice, the betatron tunes are controlled by the quadrupoles of the long straight section FODO cells. 

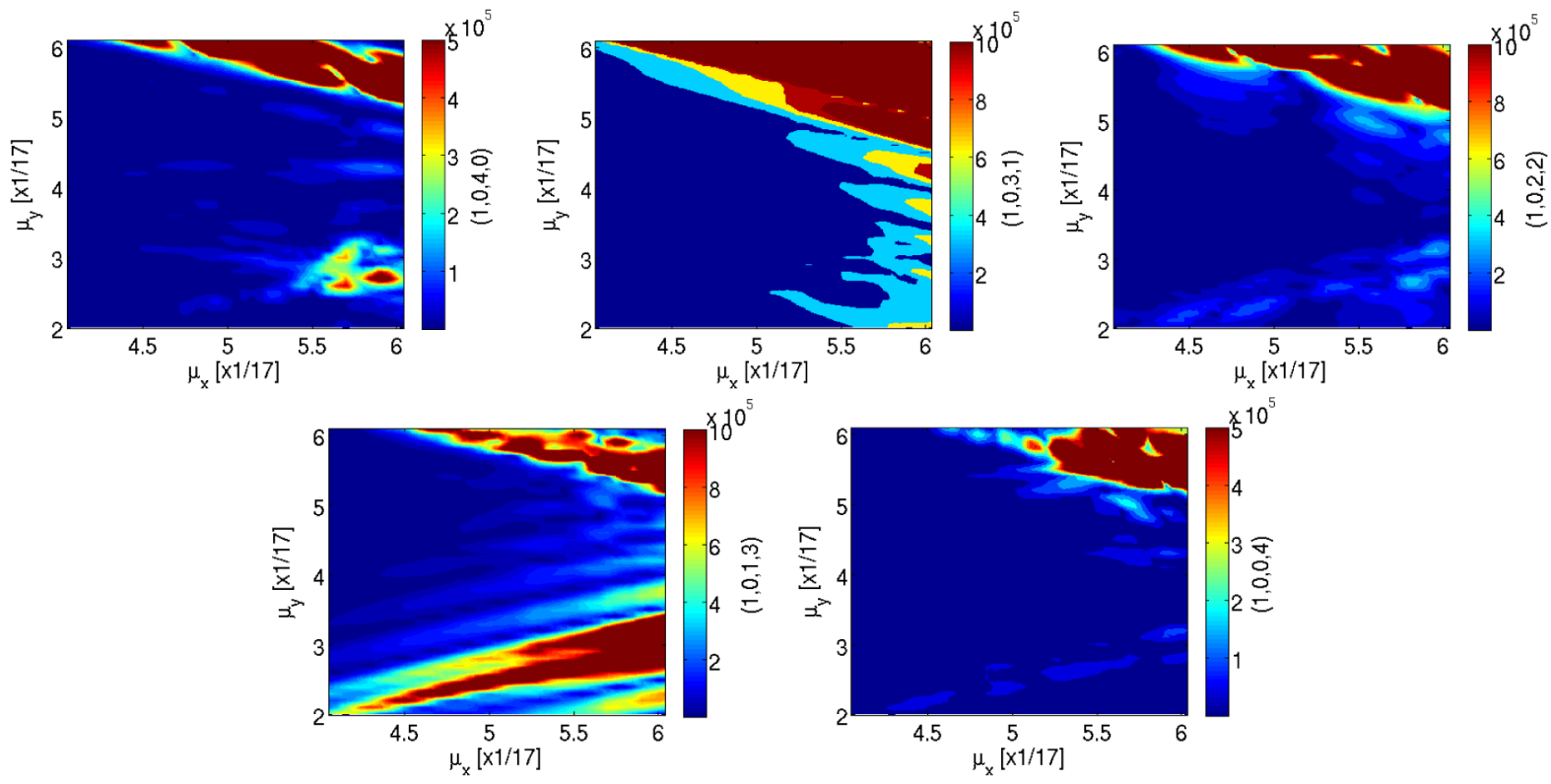

FIG. 12. The fifth order resonance driving terms for which $|j-k|=1$ and $|l-m|=4$.

Figure 14 shows the working point in tune space for momentum deviations $\delta p / p_{0}$ from $-1.2 \%$ to $1.2 \%$ (blue) and the first order tune shift with amplitude (green) up to 6 $\sigma_{x, y}$. The on-momentum working point of the linear lattice is $\left(q_{x}, q_{y}\right)=(16.39,12.26)$.

\section{B. Dynamic aperture}

The dynamic aperture (DA) is defined as the maximum phase-space amplitude within which particles do not get lost as a consequence of single-particle effects [18]. The DA has to be at least equal to or larger than the minimum beam transverse acceptance, $R_{\min }$. The beam coming from the positron source is not expected

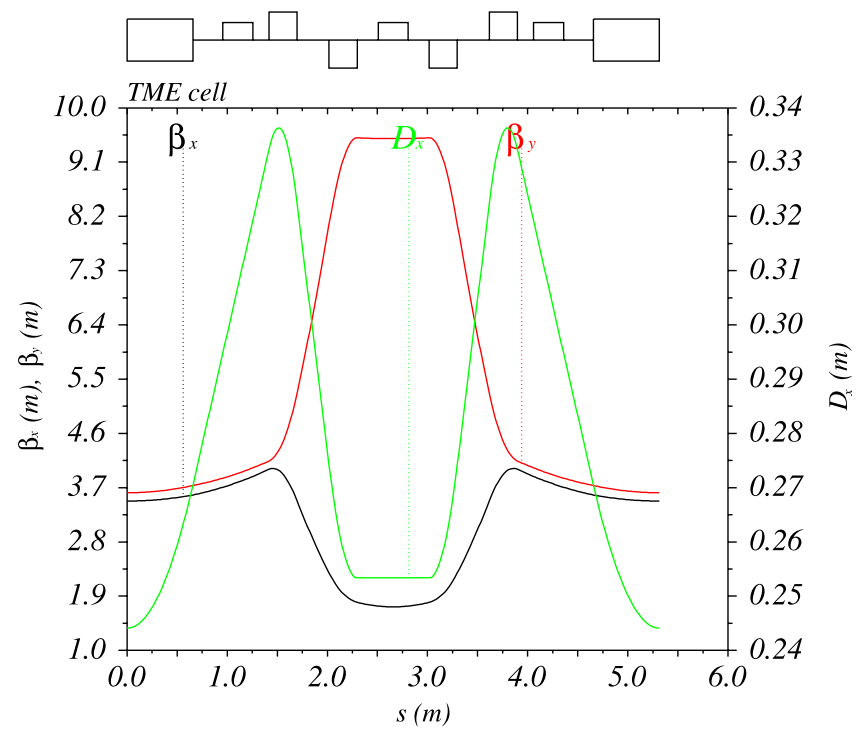

FIG. 13. The optical functions of the TME arc cell of the PDR. to be Gaussian, and the distribution in the storage ring is not modified, until the beam is damped close to equilibrium. For this reason, the minimum transverse acceptance is defined in terms of a maximum emittance $\epsilon_{\max }$ of the particles with the maximum betatron action in the beam, and of a maximum relative momentum deviation $\left(\delta p / p_{0}\right)_{\max }[8]$ :

$$
R_{\min }=\sqrt{2 \beta \epsilon_{\max }}+D\left(\delta p / p_{0}\right)_{\max }+d_{\mathrm{co}} .
$$

The incoming beam to the CLIC PDR is a round beam with same horizontal and vertical rms emittances of $\epsilon_{x, y}^{\mathrm{rms}}=$ $7 \mathrm{~mm} \mathrm{rad}$ where, $99.9 \%$ of the particles are inside a

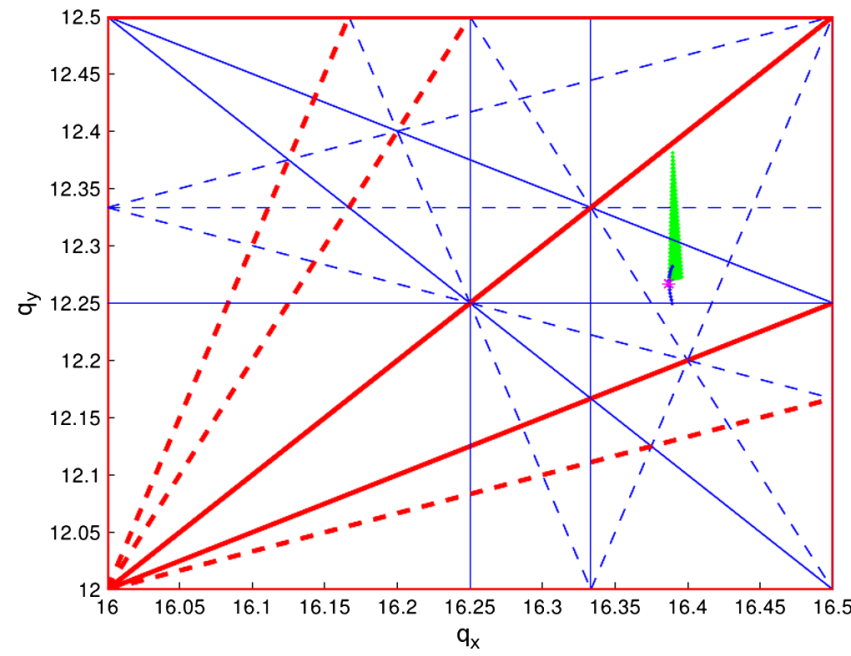

FIG. 14. The working point in tune space for $\delta p / p$ from -1.2 to $1.2 \%$ (blue) and the first order tune shift with amplitude up to $6 \sigma$ (green). The on-momentum working point is $(16.39,12.27)$. 

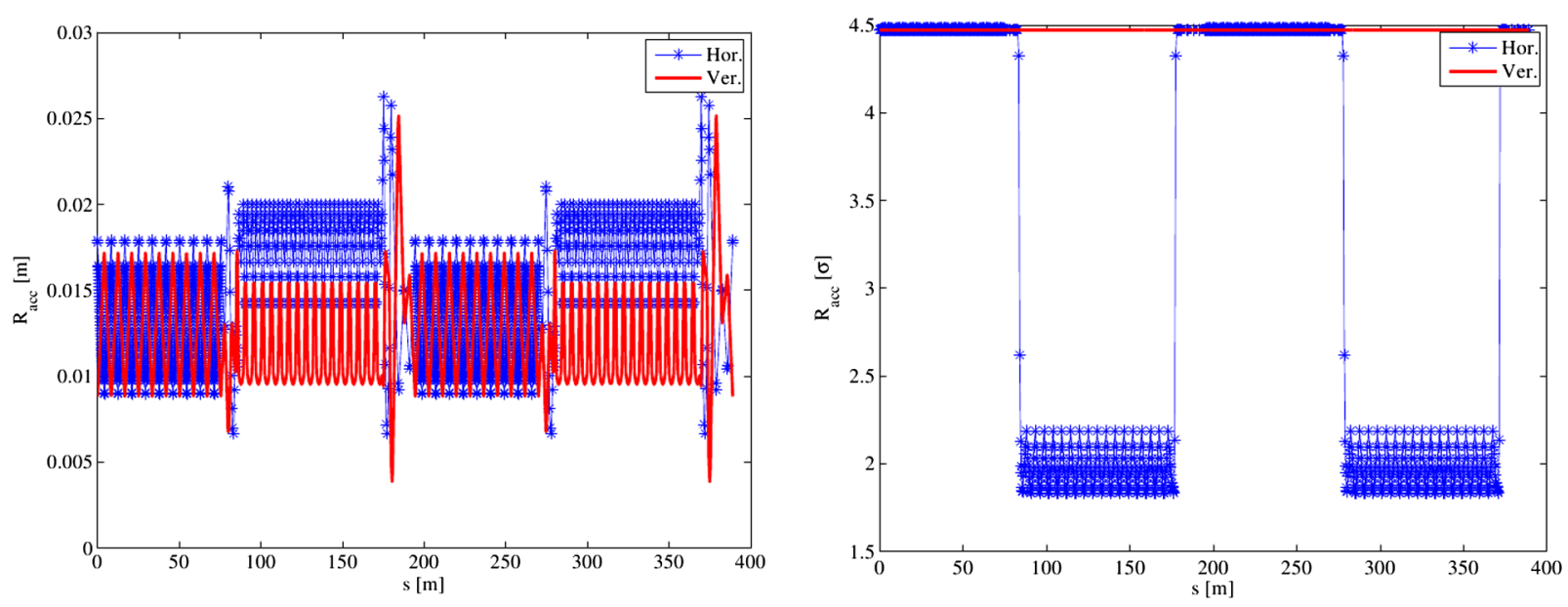

FIG. 15. The required acceptance around the PDR in order to fit the positron beam in units meters (left) and in units of beam sizes (right).

maximum emittance of $\epsilon_{\max }=10 \epsilon_{x, y}^{\mathrm{rms}}$ and with maximum $\left(\delta p / p_{0}\right)_{\max }=3 \%$. Applying this to Eq. (24), the minimum acceptance can be calculated around the ring and is shown in Fig. 15, in units of [m] (left) and in units of beam sizes $[\sigma]$ (right). A minimum DA of $4.5 \sigma_{x, y}$ is required, in both horizontal (blue) and vertical (red) planes, in order to fit the large non-Gaussian beam coming from the positron source.

The DA of the ring was computed with numerical particle tracking, over 1000 turns, with the PTC module of MADX [5]. Figure 16 shows the initial positions of particles that survived over 1000 turns, normalized to the horizontal and vertical beam sizes, at the point of calculation $\left(\sigma_{x}=4 \mathrm{~mm}, \sigma_{y}=2 \mathrm{~mm}\right)$. The results for $\delta p / p_{0}=0 \%$ are shown in red, for $\delta p / p_{0}=1.2 \%$ in green and for $\delta p / p_{0}=-1.2 \%$ in blue. The minimum acceptance is shown in black. For these calculations the magnet fringe fields are taken into account, while any magnet error effects are neglected. An adequate but tight dynamic aperture is demonstrated, following an optimization procedure based

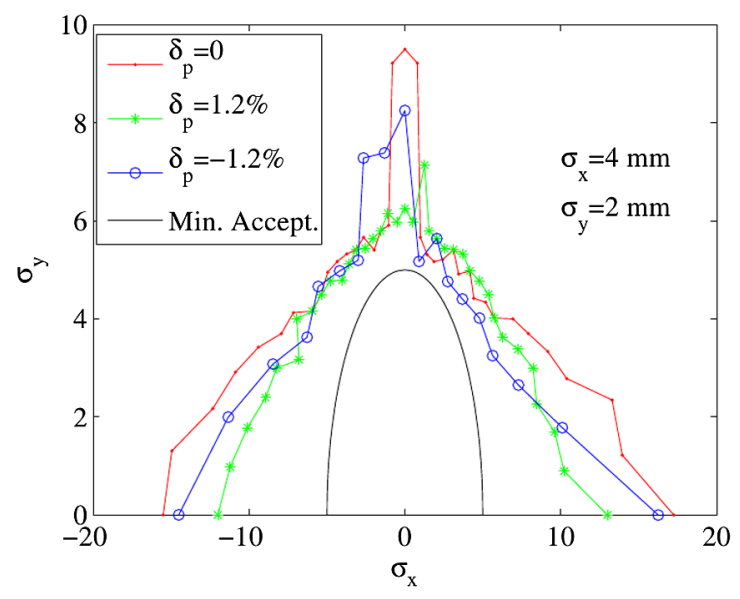

FIG. 16. The on and off momentum DA of the PDR for $\delta_{p}=0$ (red), $1.2 \%$ (green) and $-1.2 \%$ (blue). on the resonance free lattice concept, however, more optimization steps are required when magnet errors and the effect of wigglers are included.

\section{Frequency maps}

The frequency map analysis (FMA) examines the dynamics in frequency space rather than configuration space. Regular or quasiregular periodic motion is a single point in frequency space characterized by a pair of fixed tune values. Irregular trajectories exhibit diffusion in frequency space, with the tunes changing in time. The mapping of configuration space ( $x \& y$ ) to frequency space $\left(q_{x} \& q_{y}\right)$ will be regular for regular motion and irregular for chaotic motion. Numerical integration of the equations of motion, for a set of initial conditions $\left(x, y, x^{\prime}, y^{\prime}\right)$ and computation of the frequencies as a function of time (or turn number), constructs the map from the space of initial conditions to frequency or tune space, over a finite time span $T[19,20]$. An indication of how much the frequency is changing with time, is measured through the diffusion coefficient, defined by:

$$
\mathcal{D}=\log \sqrt{\left(q_{x 1}-q_{x 2}\right)^{2}+\left(q_{y 1}-q_{y 2}\right)^{2}},
$$

where the index 1 refers to a certain number of turns, while the index 2 to a consecutive same amount of turns. Large negative values of $\mathcal{D}$ denote long-term stability while values of $\mathcal{D}$ close to zero denote chaotic motion [19].

Tracking of particles with different initial conditions for 1024 turns, was performed with MADX-PTC [21]. The ideal lattice including sextupoles and fringe fields is used, while no magnet errors are taken into account. The frequency map analysis was performed with the numerical analysis of fundamental frequencies (NAFF) algorithm [19].

Figure 17 (left) shows the initial positions of particles survived over 1024 turns, color-coded with the diffusion 

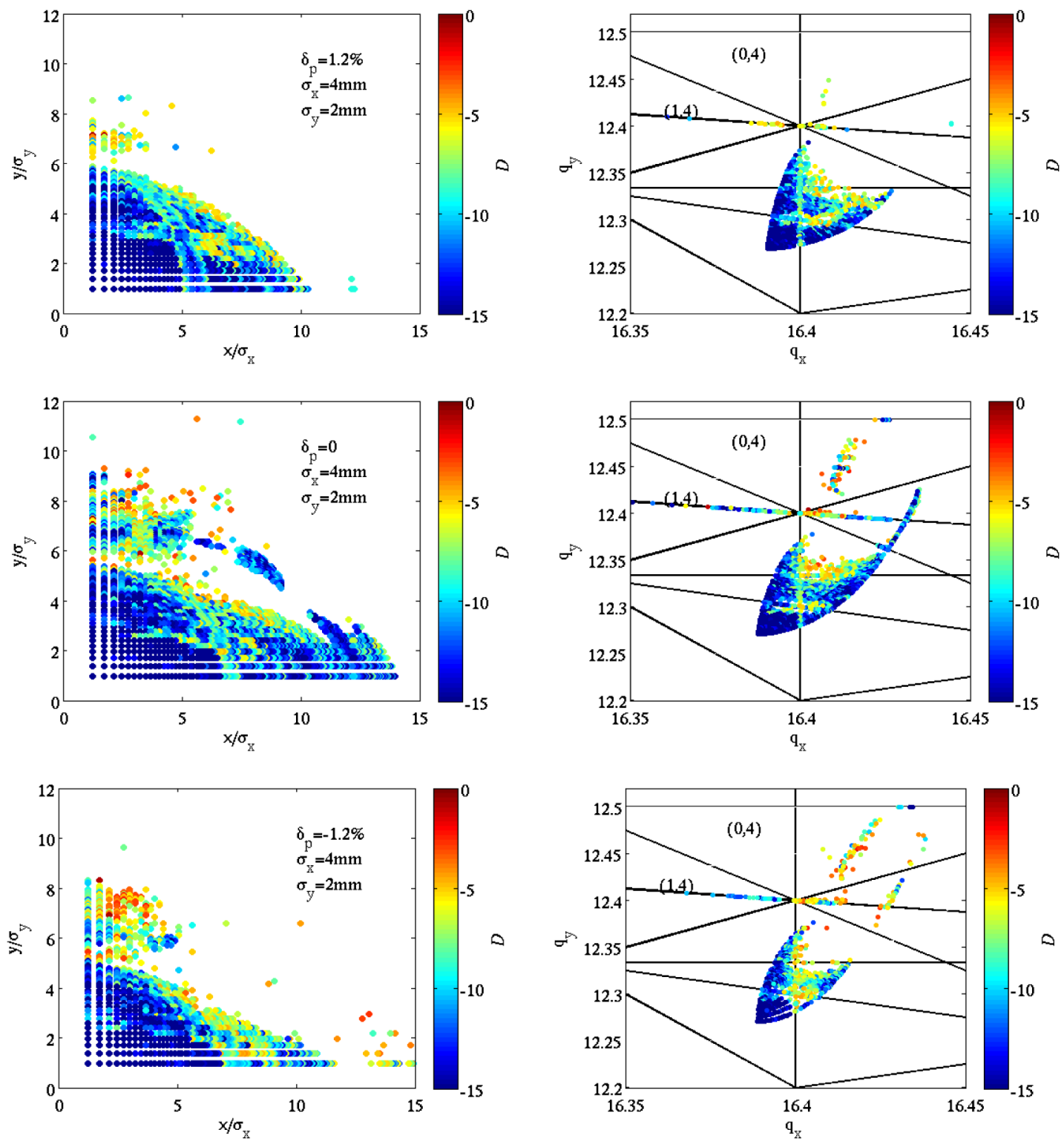

FIG. 17. Diffusion maps (left) and frequency maps (right) for $\delta p / p=1.2 \%$ (top), 0 (middle), and -1.2 (bottom).

coefficient of Eq. (25), for on-momentum particles with $\delta p / p_{0}=0 \%$ (top) and for off-momentum particles with $\delta p / p_{0}=1.2 \%$ (middle) and $\delta p / p_{0}=-1.2 \%$ (bottom). The particle positions in the horizontal and vertical axis are expressed in units of horizontal and vertical beam sizes at the point of calculation, where $\sigma_{x}=4 \mathrm{~mm}$ and $\sigma_{x}=2 \mathrm{~mm}$. The frequencies of the particles are presented in the right plots, the frequency maps. The color indicates the regularity of the orbits. Blue regions indicate very regular motion, while dark-red regions indicate chaotic motion. The absence of dots means that the particles were lost. Resonance lines in the frequency maps are shown as distorted areas, while the colors allow us to relate the resonant features observed to regions of the physical space [19]. From the frequency maps it is observed that the tune is crossing the $(1,4)$ resonance, which is not eliminated by the TME phase advance choice $\left(\mu_{x}=5 / 17, \mu_{y}=3 / 17\right)$ as shown in Fig. 12. This seems to be the main limitation of the DA.

The shape of the frequency maps, especially at high amplitudes, does not have the triangular shape expected by the linear dependence of the tune shift to the action, and they appear to be folded. This occurs when terms of higher order in the Hamiltonian become dominant over the quadratic terms as the amplitude increases [19]. This behavior occurs due to the suppression of the lower order resonances, following the resonance free lattice concept, which gives rise to higher order terms. Even though folded maps may lead to potentially very unstable designs, in our case this is not taken into account for the moment, as the folding of the map appears at high amplitudes, beyond the DA aperture limit. 


\section{CONCLUSION}

An analytical parametrization for the TME cell has been derived and presented in this paper, based on linear optics arguments and the thin lens approximation. In that way all cell properties, optical and geometrical, are globally determined and the optimization procedure following any design requirements can be performed in a systematic way. Stability criteria in both horizontal and vertical planes and magnet technology constraints are also applied. A comparison of the analytical solution with the results from the simulation code MADX gave very good agreement, even for the thick-elements optics. This method provides a very useful tool for defining optimal regions of operation for the best performance of the cell, according to the requirements of the design. The same methodology can be applied in other types of low emittance cells as well. In a double bend achromat for example, considering the part of the cell responsible for the emittance minimization (the two dipoles and the 2 symmetric quadrupoles in between) the complexity of the problem is expected to be the same. Regarding the multibend achromats, this is a combination of the TME and DBA problems. In that case, the complexity probably becomes more important, especially in the case of nonsymmetric cells. On the other hand, the optimization of single cells can be always done following the same route, as the method provides a global analytical parametrization.

The analytical approach and the resonance free lattice concept were finally used for the linear and nonlinear optimization of the CLIC predamping rings, providing an adequate dynamic aperture for a large incoming beam.

[1] S. Y. Lee and L. Teng, in Proceedings of the 1991 Particle Accelerator Conference, San Francisco, CA, 1991 (IEEE, New York, 1991), p. 2679.

[2] P. Emma and T. Raubenheimer, Phys. Rev. ST Accel. Beams 4, 021001 (2001).

[3] D. Robin, F. Sannibale, C. Steier, W. Wan, and L. Yang, in Proceedings of the 11th European Particle Accelerator Conference, Genoa, 2008 (EPS-AG, Genoa, Italy, 2008), p. 3050 .

[4] D. S. Robin, W. Wan, F. Sannibale, and V. P. Suller, Phys. Rev. ST Accel. Beams 11, 024002 (2008).

[5] MAD-X homepage, URL http://mad.web.cern.ch/mad.
[6] A. Verdier, in Proceedings of the 18th Particle Accelerator Conference, New York, 1999 (IEEE, New York, 1999), p. 398.

[7] S. Lee, Accelerator Physics (World Scientific, Singapore, 2004), 2nd ed., ISBN 9789812562005.

[8] A. Wolski, in Proceedings of the 8th European Particle Accelerator Conference, Paris, 2002 (EPS-IGA and CERN, Geneva, 2002), p. 521.

[9] Y. Papaphilippou, F. Antoniou, M. Barnes, S. Calatroni, P. Chiggiato, R. Corsini, A. Grudiev, E. Koukovini, T. Lefevre, M. Martini et al., CERN, Report No. CERN-ATS2012-176, 2012.

[10] A. Vorozhtsov, "Preliminary Design and Cost Estimate of CLIC Main Beam Normal Conducting Magnets Required for the Damping Rings" (to be published).

[11] G. Rakowsky, in Proceedings of the 2001 Particle Accelerator Conference, Chicago, Illinois (IEEE, 2001), pp. 3242-3244.

[12] Y. Jiao, Y. Cai, and A. W. Chao, Phys. Rev. ST Accel. Beams 14, 054002 (2011).

[13] O. Dadoun, I. Chaikovska, P. Lepercq, F. Poirier, A. Variola, R. Chehab, L. Rinolfi, A. Vivoli, V. Strakhovenko, and C. Xu, Technical Report No. CLIC-Note-824, 2010.

[14] H. Braun, R. Corsini, J. P. Delahaye, A. de Roeck, S. Dobert, A. Ferrari, G. Geschonke, A. Grudiev, C. Hauviller, B. Jeanneret et al., Technical Report No. CLIC-Note-764, 2008.

[15] D. Schoerling, F. Antoniou, A. Bernhard, A. Bragin, M. Karppinen, R. Maccaferri, N. Mezentsev, R. Rossmanith, G. Rumolo, S. Russenschuck et al., Phys. Rev. ST Accel. Beams 15, 042401 (2012).

[16] J. Bengtsson, Technical Report No. PSI Internal Report SLS-Note 9/97, 1997.

[17] E. Forest and J. Milutinovic, Nucl. Instrum. Methods Phys. Res., Sect. A 269, 474 (1988).

[18] A. Chao, Handbook of Accelerator Physics and Engineering (World Scientific, Singapore, 1999), ISBN 9789810235000.

[19] J. Laskar, in Proceedings of the 2003 Particle Accelerator Conference, Portland, OR (IEEE, New York, 2003), p. 378.

[20] J. Laskar, in 3DHAM'95, Proceedings of NATO Advanced Study Institutes, S'Agaro (1994), Vol. 344, pp. 130-159.

[21] F. Schmidt, C. Y. Chiu, B. Goddard, D. Jacquet, V. Kain, M. Lamont, V. Mertens, J. Uythoven, and J. Wenninger, in Proceedings of the 21st Particle Accelerator Conference, Knoxville, TN, 2005 (IEEE, Piscataway, NJ, 2005), p. 1272. 